\title{
The two $\alpha$-dox genes of Nicotiana attenuata: overlapping but distinct functions in development and stress responses
}

\author{
Anke Steppuhn ${ }^{2 \dagger}$, Emmanuel Gaquerel $^{1 \dagger}$, lan T Baldwin $^{1 *}$
}

\begin{abstract}
Background: Plant fatty acid $\alpha$-dioxygenases ( $\alpha$-DOX) are oxylipin-forming enzymes induced by biotic and abiotic stresses, which also participate in developmental processes. In Nicotiana attenuata, herbivory strongly induces the expression of an $\alpha$-dox 1 gene. To determine its role, we silenced its expression using Agrobacterium-mediated plant transformation with an inverted repeat construct. More than half of the transformed lines showed a severe dwarf growth phenotype that was very similar to the phenotype of tomato plants mutated at a second $\alpha$-dox isoform. This led us to identify the corresponding $\alpha$-dox 2 gene in N. attenuata and examine the regulation of both $\alpha$-dox genes as well as the consequences of their silencing in plant development and anti-herbivore defense.

Results: The transformed lines exhibiting a dwarf growth phenotype are co-silenced for both $\alpha$-dox genes resulting in a nearly complete suppression of $\alpha$-DOX activity, which is associated with increases in ABA, JA and anthocyanin levels, all metabolic signatures of oxidative stress. The other lines, only silenced for $\alpha$-dox 1 , developed similarly to wild-type plants, exhibited a $40 \%$ reduction of $\alpha$-DOX activity resulting in a $50 \%$ reduction of its main product in planta (2-HOT) and showed no signs of oxidative stress. In contrast to $\alpha$-dox1, the expression of $\alpha$-dox 2 gene is not induced by wounding or elicitors in the oral secretions of Manduca sexta. Instead, $\alpha$-dox 2 is expressed in roots and flowers which lack $\alpha$-dox 1 expression, but both genes are equally regulated during leaf maturation. We transiently silenced $\alpha$-dox gene copies with gene-specific constructs using virus induced gene silencing and determined the consequences for plant development and phytohormone and 2-HOT levels. While individual silencing of $\alpha$-dox 1 or $\alpha$-dox 2 had no effects on plant growth, the co-suppression of both $\alpha$-dox genes decreased plant growth. Plants transiently silenced for both $\alpha$-dox genes had increased constitutive levels of JA and ABA but silencing $\alpha$-dox1 alone resulted in lower M. sexta-induced levels of JA, 2-HOT and ABA.

Conclusions: Thus, both $\alpha$-dox isoforms function in the development of $N$. attenuata. In leaf maturation, the two $\alpha$-dox genes have overlapping functions, but only $\alpha$-dox 2 is involved in root and flower development and only $\alpha$-dox 1 functions in anti-herbivore defense.
\end{abstract}

\section{Background}

Fatty acid (FA) hydroperoxides are intermediates in different oxylipin pathways controlling plant development [1] and plant responses to stresses [2]. To date, much of the research on oxylipin signals has focused on jasmonic acid (JA) whose biosynthesis starts with the peroxidation of linolenic acid (C18:3) mediated by

\footnotetext{
* Correspondence: baldwin@ice.mpg.de

† Contributed equally

'Department of Molecular Ecology, Max-Planck-Institute for Chemical

Ecology, Hans-Knöll-Str. 8, Jena 07745, Germany

Full list of author information is available at the end of the article
}

13-lipoxygenase (13-LOX) enzymes. In Nicotiana attenuata, silencing of lox 3 , a gene coding for a 13LOX isoform, considerably reduces the accumulation of herbivory-induced jasmonic acid and as a consequence, the accumulation of several direct defense compounds, such as nicotine and trypsin proteinase inhibitors, as well as terpenoid volatiles that function as indirect defenses [3]. Consistent with its important regulatory role in mediating responses to herbivory, lox3 expression increases after herbivore attack [4].

In $N$. attenuata, one of the transcripts most strongly elicited by the feeding of different herbivores is an

\section{(Ciomed Central}


$\alpha$-dioxygenase ( $\alpha$-dox), a gene homologue of Nicotiana tabacum (Nt) $\alpha$-dox [5-8]. The Nt $\alpha-d o x$ was first identified as a pathogen inducible oxygenase (PIOX) sharing significant homologies with mammalian prostaglandin endoperoxidases [9]. $\alpha$-DOXs are FA-hydroperoxidases which target the $\alpha$-carbon (C2) of a broad range of FAs $[9,10]$. In vitro, their catalytic activity is characterized by the production of long-chain aldehydes formed from $\alpha$-hydroperoxy-FA molecules that escape reduction to $\alpha$-hydroxy-FA and undergo spontaneous decarboxylation [10]. Like LOXs, $\alpha$-DOXs also use linolenic acid (C18:3) as substrate, but convert it to heptadecatrienal (HDT) in vitro. However, quantification of the oxidative products of $\alpha$-DOX activity in response to bacterial inoculation, revealed that 2-hydroxy-C18:3 (2-HOT) is the major product synthesized in tobacco plants [11]. Because 2-HOT exhibits antimicrobial activity at high concentrations, it may directly protect plants against pathogen attack $[11,12]$. However, the biological functions of this and other $\alpha$-DOX products remain largely unknown.

Several lines of evidence indicate that $\alpha$-dox genes are involved in responses to different abiotic and biotic stresses. In tomato roots, for example, ethylene increases $\alpha$-dox transcript accumulation during salt stress [13]. Other abiotic stresses, such as UV-B exposure, heavy metal stress, and cold stress, increase $\alpha$-dox transcript accumulation in Nicotiana longiflora, rice and Arabidopsis, respectively [14-16]. However, more work has examined whether $\alpha$-DOX is involved in a plant's response to pathogens and herbivores. In Capsicum annuum transcripts of an $\alpha$-DOX homolog increase during pathogen infection [17]. In Arabidopsis thaliana and N. tabacum, the transcriptional up-regulation of $\alpha$-dox is amplified when the infection results in a hypersensitive response $[9,18]$. Ponce de León et al. [19] showed that $\alpha$-dox gene expression is impaired in salicylic acid (SA)compromised plants and transgenic A. thaliana plants silenced in $\alpha$-dox expression do more rapidly develop severe necrotic lesions in response to incompatible bacteria than do wild-type (WT) plants. This suggests that $\alpha$-DOX1 activity protects tissues from excessive necrosis; however, the responsible mechanisms remain unknown.

In $N$. attenuata, the effects of pathogen inoculation and treatment with pathogen-derived elicitors on $N$. attenuata $\alpha$-dox transcript accumulation is much weaker than that of its homologues in other species [5]. The strong transcriptional up-regulation of $N$. attenuata $\alpha$-dox in response to herbivore attack involves the initial perception of fatty acid amino acid conjugates (FACs), which are herbivore specific elicitors in the oral secretions (OS) of lepidopteran larvae [20]. In addition, the up-regulation of $\alpha$-dox requires the JA-signaling pathway as demonstrated by the lack of $\alpha$-dox transcript accumulation in OS-elicited lox3-silenced plants [3]. JA elicitation of $\alpha$-dox transcripts has also been reported in Oryza sativa [15] and N. tabacum [9]. This regulation suggests an anti-herbivore function for $\mathrm{Na} \alpha-\mathrm{DOX}$ in N. attenuata.

Generally, the increased transcription of $\alpha$-dox genes in plants attacked by herbivores and pathogens suggests a defensive function; however, plant $\alpha$-dox transcripts and activity are also regulated during developmental processes. Fatty acid $\alpha$-oxidation was first reported in peanut seedlings during germination [21]. Also during germination, one of the first $\alpha$-DOX proteins was isolated in pea. Pea seedlings accumulate $\operatorname{Ps} \alpha-d o x$ transcripts at much higher levels than do leaves and dry seeds [22] and these are lost during maturation [23] and later detected exclusively in roots. Additional evidence for a role in development is suggested by the increasing expression $\mathrm{Nt} \alpha$-dox gene during leaf senescence in N. tabacum [24].

FA $\alpha$-oxidation is phylogenetically widespread, as even preparations from green alga form 2-hydroxypalmic acid from palmic acid [25]. The $\alpha$-DOX proteins identified in different plant species show high amino acid homologies. For example, the amino acid sequence of $N$. tabacum $\alpha$-DOX shares high amino acid similarities with the proteins in $N$. attenuata (95\% identity), C. annuum (85\% identity), Solanum lycopersicum (84\% identity), A. thaliana (75\% identity), and O. sativa (63\% identity) [18]. In addition to these proteins, referred to as $\alpha$-DOX1, another $\alpha$-DOX isoform exists. A second protein, first identified in tomato and $A$. thaliana, shares more homology between species than with the corresponding $\alpha$-DOX1 sequence of the same species. This second protein, encoded by $\alpha$-dox2, is the S. lycopersicum feebly gene, which leads to a dwarf phenotype in a knock-out mutant [26]. The patterns of $\alpha$ dox 2 transcript accumulation differ from those of $\alpha$-dox 1 in both S. lycopersicum and A. thaliana. Whereas $\alpha$-dox2 transcript accumulation is not increased by pathogen infection, it is enhanced in seedlings and in wilting leaves 3 to 6 days after detachment [27]. Despite a similar transcriptional regulation of $\alpha$-dox2 in S. lycopersicum and $A$. thaliana, its role in plant development appears to be species-specific. Ectopic expression of the At $\alpha-d o x 2$ in a tomato mutant deficient for $\alpha$-dox 2 partially complemented the compromised growth phenotype, but deletion of At $\alpha$-dox 2 did not result in a growth phenotype [28]. In summary, although $\alpha$-DOX proteins are widespread across the plant kingdom their specific functions, presumably in defense and development, remain to be elucidated.

To determine the role of the herbivore responsive $\alpha$-dox 1 gene in $N$. attenuata, we used Agrobacterium mediated plant transformation with an inverted repeat (IR) construct to silence its expression. More than half 
of the lines showed a severe dwarf growth phenotype, the other lines developed similarly to WT plants. Though the $\alpha$-DOX activity was reduced in all lines, it was not detectable in those lines exhibiting a dwarf phenotype. To test whether this resulted from a co-silencing of a second $\alpha$-dox isoform in the dwarf phenotype lines, we used a consensus sequence of $\alpha$-dox2 isoforms in L. esculentum and $A$. thaliana to clone a gene fragment of the $N$. attenuata $\alpha$-dox 2 gene. The dwarf phenotype correlated with the co-silencing of the $\mathrm{Na} \alpha-d o x 2$ gene. In contrast to $\mathrm{Na} \alpha$-dox $1, \mathrm{Na} \alpha$-dox 2 transcripts were not induced by wounding or simulations of Manduca sexta attack. Instead, $\alpha-d o x 2$ was more expressed in roots and flowers. To investigate the functions of both isoforms, we transiently silenced both with gene specific constructs using virus induced gene silencing (VIGS) and examined the consequences for plant development and constitutive and induced phytohormone levels.

\section{Results}

\section{Silencing $\mathrm{Na} \alpha$-dox in N. attenuata results in two growth} phenotypes

To examine the function of Na $\alpha$-dox1 [GenBank AF229926], we transformed $N$. attenuata with an IR construct containing a $457 \mathrm{bp}$ fragment of $\mathrm{Na} \alpha-\operatorname{dox} 1$ to silence its expression (Additional file 1). Two distinct growth phenotypes were observed: 11 of the 18 independently transformed lines showed stunted growth $\left(\operatorname{IR}_{\alpha-d o x} S\right)$, whereas the other lines showed a mild phenotype $\left(\mathrm{IR}_{\alpha-d o x} \mathrm{M}\right)$ and were indistinguishable from WT plants in their growth and development. Plants of the $\mathrm{IR}_{\alpha-d o x} \mathrm{~S}$ lines germinate normally though a higher proportion of infertile seeds were produced by these plants. Young $\mathrm{IR}_{\alpha-\text { dox }} \mathrm{S}$ seedlings appear similar to WT plants, but already when transplanted into soil two weeks after germination, they are slightly delayed in development. At rosette stage, approximately 35 days after germination, $\mathrm{IR}_{\alpha-d o x} \mathrm{~S}$ plants are clearly delayed with rosette diameters of about a third of that of WT and $\mathrm{IR}_{\alpha-d o x} \mathrm{M}$ plants (Figure 1A). At flowering, approximately 60 days after germination, $\mathrm{IR}_{\alpha-d o x} \mathrm{~S}$ plants only attained one fourth to a third of the height of WT plants and produced only few flowers (Figure 1B). Whereas WT $N$. attenuata plant in $1 \mathrm{~L}$ pots produce between 50 and 120 capsules, homozygous $\mathrm{IR}_{\alpha-d o x} \mathrm{~S}$ plants usually produce between 0 and 5 capsules. The $\alpha$-DOX activity was reduced in all $\mathrm{IR}_{\alpha-d o x}$ lines. However, whereas $\mathrm{IR}_{\alpha-}$ ${ }_{d o x} \mathrm{M}$ plants showed a reduction of around $40 \%$ in $\alpha$-DOX activity, only very low or no activity was detectable in dwarf $\mathrm{IR}_{\alpha-d o x} \mathrm{~S}$ plants (Figure $1 \mathrm{C}$ ). The accumulation of the main product of $\alpha$-DOX in planta: $2-\mathrm{HOT}$ was reduced by more than $50 \%$ in $\mathrm{IR}_{\alpha-d o x} \mathrm{M}$ plants compared to WT and was at the detection limit in $\mathrm{IR}_{\alpha-d o x} \mathrm{~S}$ plants (Figure 1D).

\section{A $\alpha$-dox 2 isoform in $N$. attenuata is co-silenced in the $\mathrm{IR}_{\alpha \text {-dox }}$ lines}

In $S$. lycopersicum and $A$. thaliana a second gene with high sequence similarity to $\alpha$-dox 1 was identified [27]. The phenotype of tomato plants mutated at this $\operatorname{Sl} \alpha$ dox2 locus (feebly, divarcata) is very similar to that of the $\operatorname{IR}_{\alpha-d o x} S$ plants $[26,28]$. A Southern-blot analysis performed by Hermsmeier et al. (2001) has already demonstrated that $N$. attenuata's genome contains at least two $\alpha$-dox gene copies. We therefore investigated whether a homologue of $S$. lycopersicum and A. thaliana $\alpha$-dox2 may also be present in $N$. attenuata and may have been co-silenced in the $\mathrm{IR}_{\alpha \text {-dox }} \mathrm{S}$ plants. We used a consensus sequence of $\alpha$-dox 2 isoforms in S. lycopersicum and A. thaliana with low sequence identity to the N. attenuata $\alpha$-dox 1 gene to design primers to amplify a putative $\mathrm{Na} \alpha$-dox 2 gene. We amplified a sequence and cloned it into Escherichia coli. Subsequently, a 355 bp gene fragment [GenBank EU681953.1] of the $\mathrm{Na} \alpha$-dox2 was sequenced from 3 independent clones. By comparison of this gene fragment to a custom data base of the complete $N$. attenuata transcriptome we identified a cDNA sequence of Na $\alpha$-dox 2 of 2290 bp [GenBank HM140643]. The translated amino-acid sequence deduced from this sequence clusters in a phylogenetic tree of plant $\alpha$-DOX sequences close to the $\alpha$-dox 2 isoforms in S. lycopersicum and A. thaliana but apart from $N$. attenuata $\alpha$-dox 1 (Additional file 2).

By SYBR Green-based real-time (RT)-PCR with gene specific primers for $\mathrm{Na} \alpha$-dox 1 and $\mathrm{Na} \alpha$-dox 2 (for primer specificity see Additional file 3A), transcript accumulation of both genes was determined in $\mathrm{IR}_{\alpha-d o x} \mathrm{M}$ and $\mathrm{IR}_{\alpha-}$ ${ }_{d o x} \mathrm{~S}$ plants after repeated treatment with $M$. sexta OS to puncture wounds, a treatment known to strongly induce $\mathrm{Na} \alpha$-dox 1 transcription [29]. Transcript accumulation of $\mathrm{Na} \alpha$-dox 1 was reduced by $40 \%$ in $\mathrm{IR}_{\alpha-d o x} \mathrm{M}$ and by $85 \%$ in $\mathrm{IR}_{\alpha-\text { dox }} \mathrm{S}$ plants of WT levels (Figure $2 \mathrm{~A}$ ). Moreover, in $\mathrm{IR}_{\alpha-\text { dox }} \mathrm{S}$ plants transcript accumulation of $\mathrm{Na} \alpha-d o x 2$ transcripts was reduced by $70 \%$, whereas $\mathrm{IR}_{\alpha-\text { dox }} \mathrm{M}$ plants had $\mathrm{Na} \alpha$-dox 2 transcript levels comparable to those of WT plants (Figure 2B). Thus, the dwarf phenotype of $\mathrm{IR}_{\alpha-d o x} \mathrm{~S}$ plants is correlated with a concomitant silencing of the $\mathrm{Na} \alpha$-dox 2 isoform in conjunction with a stronger silencing of the $\mathrm{Na} \alpha-\operatorname{dox} 1$ gene.

\section{Distinct transcriptional regulation of $\mathrm{Na} \alpha$-dox 1 and $\mathrm{Na} \alpha$ - dox2}

To evaluate the roles that $\mathrm{Na} \alpha$-dox 1 and $\mathrm{Na} \alpha$-dox 2 play during development we analyzed transcripts of both genes in various tissues of developing $N$. attenuata plants. Both genes show strong tissue specific differences in their transcript abundance (Figure 3A). In N. attenuata leaves, transcripts of both Na $\alpha$-dox genes accumulate with plant and leaf maturation resulting in highest 

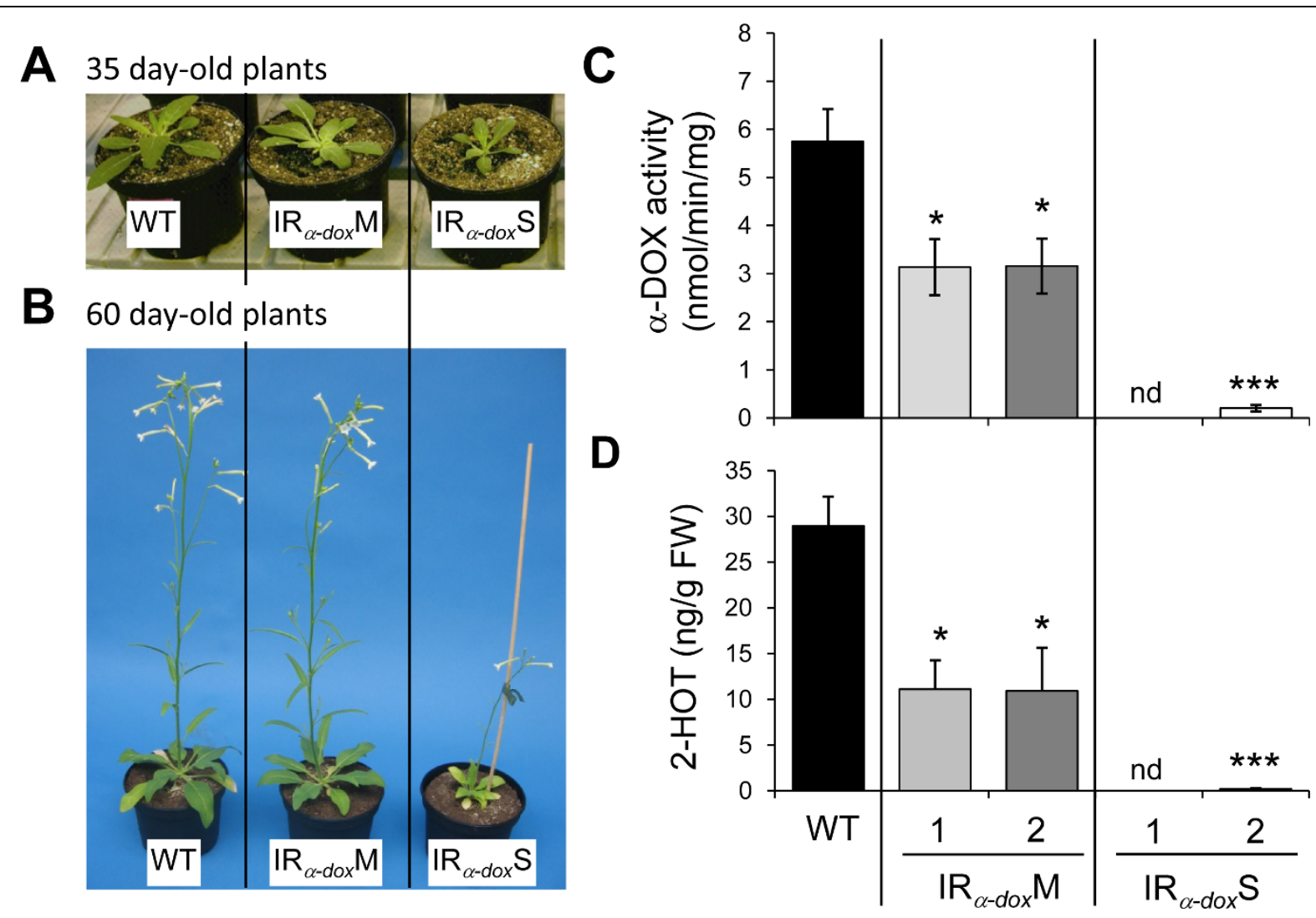

Figure 1 Silencing Na $\alpha$-dox 1 results in lines with two different growth phenotypes correlating with the suppression level of $\alpha$-DOX activity. The growth phenotype of (A) 35-day-old and (B) 60-day-old WT and $T_{3}$ homozygous Nicotiana attenuata plants transformed with an inverted repeat (IR) construct to silence Na $\alpha$-dox1. More than half of 18 independently transformed lines are severely stunted $\left(I R_{\alpha \text {-dox }} S\right)$ whereas the other lines $\left(\mathrm{IR}_{\alpha \text {-dox }} \mathrm{M}\right)$ are morphologically indistinguishable from wild-type plants (WT). (C) $\alpha$-DOX activity (mean \pm SE of 4 biological replicates) of protein extracts from leaves by in vitro measurements of the formation of 8,11,14-heptadecatrienal (HDT). Leaves had been wounded and treated with $2.5 \mu \mathrm{L}$ of OS $\mathrm{WT}_{\mathrm{T}}$ every $30 \mathrm{~min}$ for four times. Leaves were harvested $30 \mathrm{~min}$ after the last treatment. (D) Leaf levels of 2-hydroxyoctadetrienoic acid (2-HOT; mean \pm SE of 4 biological replicates), the main $\alpha$-DOX product in planta. Asterisks signify significant differences between $\mathbb{R}_{\alpha \text {-dox }}$ and $W T$ plants (unpaired t-test WT vs. lines $P<0.05$ ). nd: not detected.

transcript levels in senescing leaves. In leaf tissues, $\mathrm{Na} \alpha$ dox 1 transcripts are generally more abundant than $\mathrm{Na} \alpha-$ dox 2 transcripts. Though this difference was only significant in the young rosette leaves, the same tendency was observed in stem leaves, rosette leaves (paired $t$-test $\mathrm{P}=$ 0.053), and senescing leaves. The difference between the two $\mathrm{Na} \alpha-d o x$ genes was more pronounced in roots, flower buds, and flowers, but in these tissues $\mathrm{Na} \alpha$-dox 2 transcripts were much more abundant than those of $\mathrm{Na} \alpha$-dox1. In brief, $\mathrm{Na} \alpha$-dox 2 expression is increased in roots, during flower formation, as well as during leaf maturation and senescence, but only the latter process also enhanced $\mathrm{Na} \alpha$-dox 1 transcripts.

As both $\mathrm{Na} \alpha-$ dox genes have a similar transcriptional regulation during normal leaf maturation, the question arises, whether the $\mathrm{Na} \alpha-d o x 2$ is also regulated during herbivore attack as is known for the $\mathrm{Na} \alpha$-dox 1 gene in the leaves of $N$. attenuata. Therefore, we compared the inducibility of $\mathrm{Na} \alpha-d o x 1$ and $\mathrm{Na} \alpha$-dox 2 transcript accumulation by wounding and herbivore specific elicitors. By SYBR Green-based RT-PCR gene specific transcript accumulation of $\mathrm{Na} \alpha-d o x 1$ and $\mathrm{Na} \alpha$-dox 2 genes was determined in WT $N$. attenuata plants after repeated treatment with $M$. sexta OS to puncture wounds. As known from previous studies $[5,30]$ transcript accumulation of $\mathrm{Na} \alpha$-dox 1 is increased significantly by wounding and is further up-regulated by the treatment of the wounds with $M$. sexta OS (Figure 3B). Interestingly, the $\mathrm{Na} \alpha-d o x 2$ gene does not respond to wounding or to the application of $M$. sexta OS.

\section{Co-silencing of both $\mathrm{Na} \alpha$-dox genes increases constitutive} levels of $A B A, J A$ and anthocyanins

We measured phytohormone levels in leaves to characterize the metabolic consequences of silencing either $\mathrm{Na} \alpha-d o x 1$ or additionally $\mathrm{Na} \alpha-d o x 2$. We quantified levels of JA, abscisic acid (ABA) and SA because previous studies indicated that $\alpha$-DOX is involved in the 


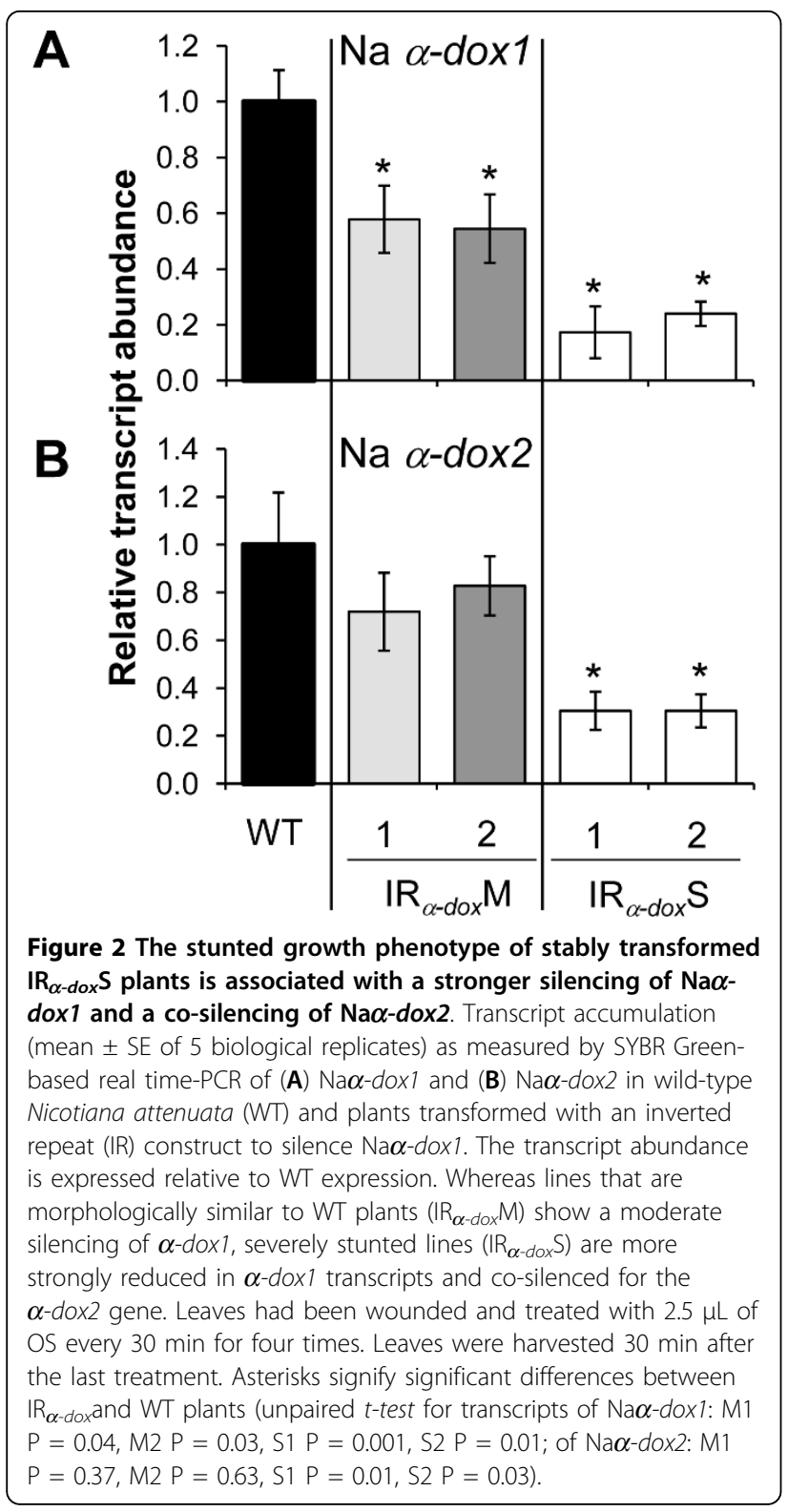

physiological processes mediated by these phytohormones. The major signaling molecule in herbivore induced responses, JA, has been shown to be required for $\alpha$-dox 1 expression in $N$. attenuata [3] and levels of ABA and SA have been reported to regulate $\alpha$-dox expression in Arabidopsis and tomato plants $[9,13]$. The $\mathrm{IR}_{\alpha-d o x} \mathrm{~S}$ plants that were co-silenced for both $\mathrm{Na} \alpha$-dox isoforms showed strongly increased levels of the phytohormone $\mathrm{ABA}$, but the $\mathrm{IR}_{\alpha-d o x} \mathrm{M}$ plants did not (Additional file 4A). In 39-days old rosette stage plants, we found an almost 3-fold increase in constitutive ABA levels and a slight increase in constitutive JA levels (Additional file $4 \mathrm{~B}$ ) in $\mathrm{IR}_{\alpha-d o x} \mathrm{~S}$ plants compared to WT. Similarly, constitutive levels of SA were increased in $\mathrm{IR}_{\alpha-d o x} \mathrm{~S}$ plants compared to WT (Additional file 4C).
Because the retarded growth phenotype of the $\mathrm{IR}_{\alpha-}$ ${ }_{d o x} \mathrm{~S}$ plants is already evident at the seedling stage, we also measured anthocyanins in the seedling stage. Anthocyanins are general indicators of stress responses and thought to be involved in a stress signaling response via reactive oxygen species (ROS) [31]. In seedlings of an $\mathrm{IR}_{\alpha \text {-dox }} \mathrm{S}$ line, levels of total anthocyanins were more than five-fold higher than WT levels 11 and 15 days after germination started (Additional file 3B). Seedlings of a second $\mathrm{IR}_{\alpha-d o x} \mathrm{~S}$ line had anthocyanin levels increased by more than $10 \% 19$ days after sowing. The increase in anthocyanins in $\mathrm{IR}_{\alpha \text {-dox }} \mathrm{S}$ lines compared to WT seedlings was significant (MannWhitney $U$-tests $\mathrm{P}<0.05$ for 11 and 15 days old seedlings of both lines and for 19 days old seedling of one line).

\section{Consequences of silencing Na $\alpha$-dox 1 and Na $\alpha$-dox 2 individually and concomitantly}

To disentangle the single and combined effects of $\mathrm{Na} \alpha$ dox 1 and $\mathrm{Na} \alpha$-dox 2 silencing we created gene-specific silencing constructs and transiently silenced $\mathrm{Na} \alpha-d o x 1$ and $\mathrm{Na} \alpha$-dox 2 separately and in combination using VIGS and examined growth parameter. That VIGS constructs were gene specific was confirmed by real time PCR for Na $\alpha$-dox 1 and Na $\alpha$-dox 2 genes in VIGS plants (Figure 4A). To compare VIGS of Na $\alpha$-dox 1 with stably transformed in $\mathrm{IR}_{\alpha-d o x} \mathrm{M}$ lines and to $\mathrm{IR}_{\alpha-d o x} \mathrm{~S}$ lines we inoculated in addition to WT also $\mathrm{IR}_{\alpha-d o x} \mathrm{M}$ plants with the empty vector (ev) and the VIGS construct to specifically silence $\mathrm{Na} \alpha-d o x 2$. Stable and transient silencing of only $\mathrm{Na} \alpha-d o x 1$ as well as transient silencing of only $\mathrm{Na} \alpha-d o x 2$ did not affect plant growth, but the additional silencing of $\mathrm{Na} \alpha-d o x 2$ in a stable line silenced for $\mathrm{Na} \alpha$ dox1 resulted in stunted growth (Figure 4B, C). Thus, VIGS $\alpha$-dox 2 in $\mathrm{IR}_{\alpha \text {-dox }} \mathrm{M}$ transformants resembled the growth phenotype when both genes were co-silenced in stably transformed $\mathrm{IR}_{\alpha-d o x} \mathrm{~S}$ lines.

To investigate the role of $\mathrm{Na} \alpha-d o x$ genes in the plant response to herbivory, we analyzed phytohormones regulating physiological processes that $\alpha-\mathrm{DOX}$ has been associated with and induced nicotine accumulation in the plants transiently silenced for either $\mathrm{Na} \alpha-d o \times 1$ or $\mathrm{Na} \alpha-$ dox 2 or both. Further, we accessed the contribution of $\mathrm{Na} \alpha-d o x 1$ and $\mathrm{Na} \alpha-d o x 2$ to constitutive and M. sextainduced 2-HOT production. Constitutive 2-HOT levels were significantly reduced in plants silenced for $\mathrm{Na} \alpha$-dox 1 or $\mathrm{Na} \alpha$-dox2 (Figure 5A) with the strongest reduction in plants silenced for both genes. Herbivory by $M$. sexta significantly increased 2-HOT levels. This induction was compromised in all plants silenced in $\mathrm{Na} \alpha$-dox 1 (Fisher's PLSD VIGSev in WT vs. $\alpha$-dox 1 silenced transformants $\mathrm{P}<0.001$ ) but silencing only $\mathrm{Na} \alpha-d o x 2$ accounted for a minor reduction in herbivory-induced 2-HOT (Fisher's 
A

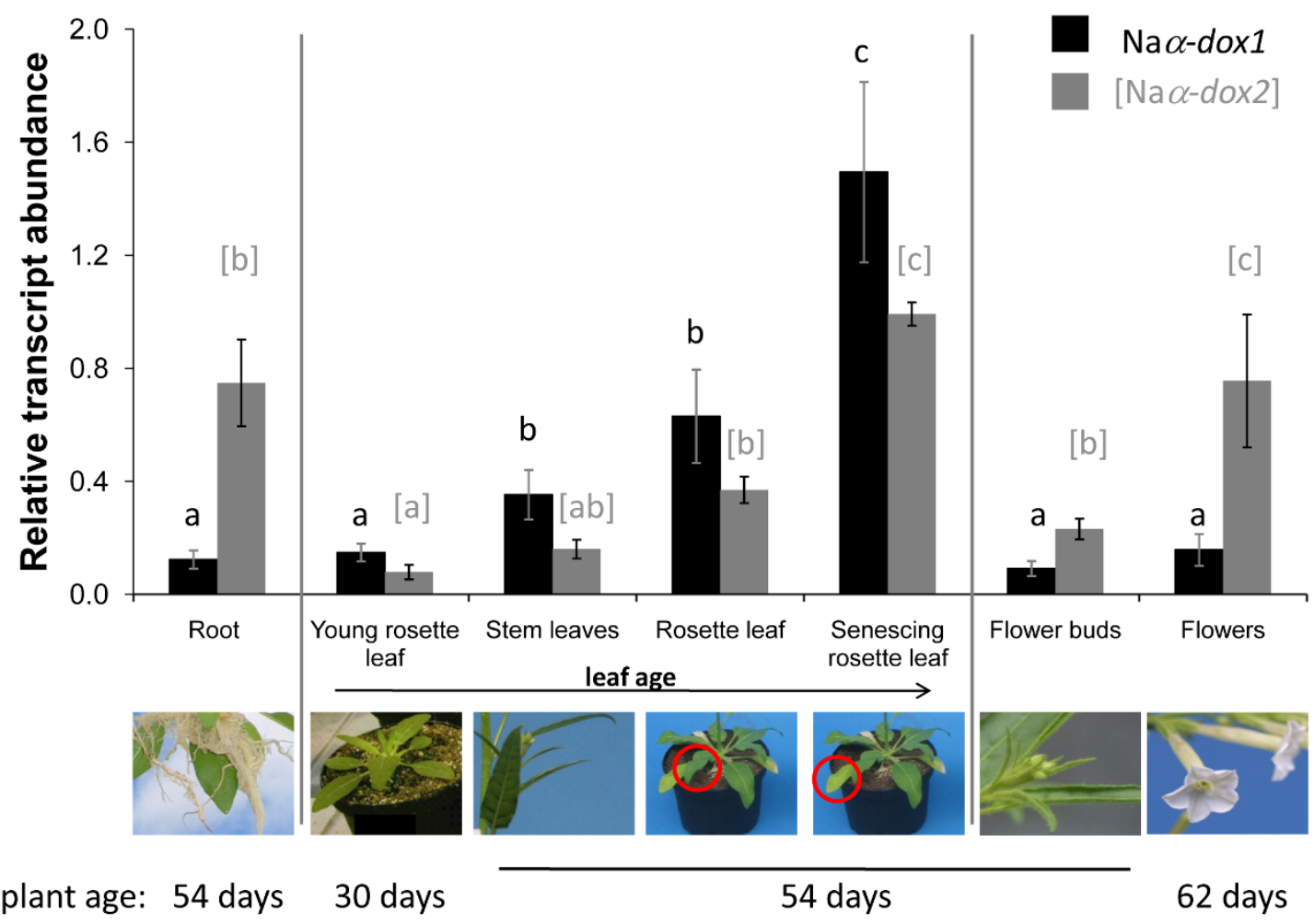

B

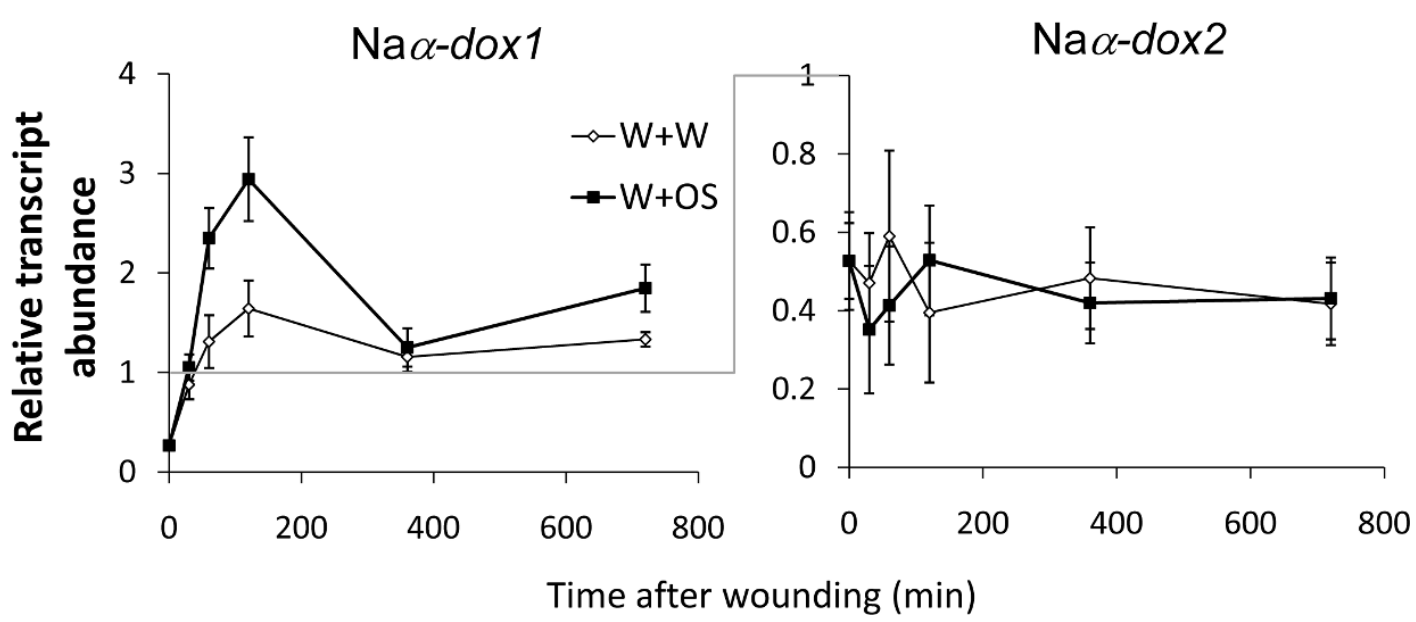

Figure 3 Transcriptional regulation of $\mathrm{Na} \alpha$-dox genes in development and the response to wounding and Manduca sexta oral secretions. (A) Mean \pm SE (3 biological replicates) of transcript abundance of Na $\alpha$-dox 1 and Na $\alpha$-dox2 (relative to actin transcripts) in various tissues of developing wild-type Nicotiana attenuata. Different letters signify significant differences between different tissues for transcript abundance of Na $\alpha$-dox1 (in black) and Na $\alpha$-dox2 (in gray and brackets) respectively (ANOVAs with factor tissue: $\alpha$-dox1 $\mathrm{F}_{6,21}=18.65, \mathrm{P}<0.0001$ and $\alpha$-dox2 $\mathrm{F}_{6,21}=20.63, \mathrm{P}<0.0001$; Fisher's PLDS for significant differences $\mathrm{P}<0.036$ ). Paired t-tests were used to test for significant differences between transcript abundance of Na $\alpha$-dox 1 and Na $\alpha$-dox 2 in the tissues (roots: $P=0.005$, young rosette leaves $P=0.047$, stem leaves $P=0.053$, old rosette leaves $P=0.047$, senescing leaves $P=0.075$, flower buds $P=0.02$, flowers $P=0.03$ ). (B) Mean $\pm S E$ (5 biological replicates) of transcript abundance of $\mathrm{Na} \alpha$-dox 1 and $\mathrm{Na} \alpha$-dox2 (relative to actin transcripts) in leaves from $\mathrm{N}$. attenuata rosette stage plants wounded at the first fully expanded source leaf with a fabric pattern wheel every $30 \mathrm{~min}$ for four times. To the resulting puncture wounds $2.5 \mu \mathrm{L}$ of either water (W) or M. sexta's oral secretions (OS) were added. Only the expression of Na $\alpha$-dox 1 but not that of Na $\alpha$-dox 2 increased after wounding, which was amplified by OS application (2 factorial ANOVA with factors treatment and time: Na $\alpha$-dox $1 \mathrm{P}<0.0046$ for both factors the interaction; for Na $\alpha$-dox2: P > 0.45 for both factors the interaction). 


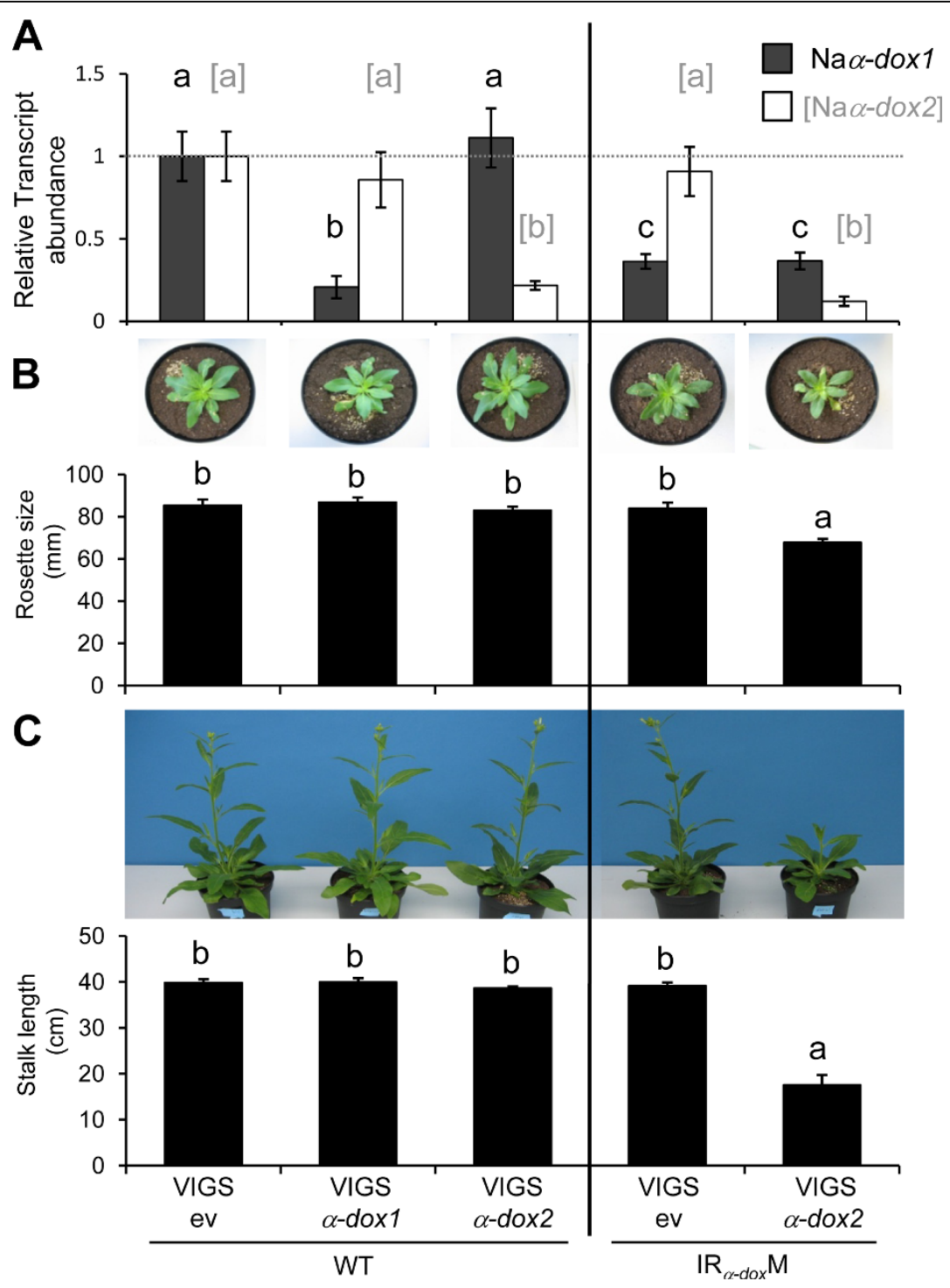

Figure 4 Co-silencing of Na $\alpha$-dox 1 and Na $\alpha$-dox 2 reduced plant growth of Nicotiana attenuata. (A) Relative transcript abundance of Na $\alpha-$ dox genes (mean \pm SE of 5 biological replicates) in plants subjected to virus-induced gene silencing (VIGS). Wild-type plants (WT) and plants stably silenced for Na $\alpha$-dox 1 using an inverted repeat (IR) construct were inoculated with VIGS constructs when 22 days old. Gene expression levels relative to empty vector controls (ev) confirm the gene specific silencing of Na $\alpha$-dox 1 and $\mathrm{Na} \alpha$-dox 2 and was analyzed in rosette leaves by SYBR Green-based real time PCR (B) Rosette diameter of 39-days old and (C) stalk length of 60-days old plants (mean \pm SE of 11 biological replicates) silenced for $\mathrm{Na} \alpha$-dox1 and Na $\alpha$-dox2 by VIGS. Stable and transient silencing of only Na $\alpha$-dox 1 as well as transient silencing of only $\mathrm{Na} \alpha$-dox2 did not affect plant growth, but the silencing of $\mathrm{Na} \alpha$-dox 2 in a stable line silenced for Na $\alpha$-dox 1 resulted in stunted growth. Different letters signify significant differences in growth parameters or transcript abundance of Na $\alpha$-dox1 (in black) and Na $\alpha$-dox2 (in gray and brackets) respectively (ANOVAs with factor transformant: for rosette diameters $F_{4,50}=12.81, P<0.0001$ for stem length $F_{4,50}=197.87, P<0.0001$; Fischer's $P L S D$ comparisons between VIGS $\alpha$-dox2 in $\mathbb{R}_{\alpha \text {-dox }} \mathrm{M}$ vs. all other transformants $\mathrm{P}<0.0001$, whereas for comparisons between all other transformants $P>0.21$.

PLSD VIGS $\alpha$-dox2 in WT vs. all transformants silenced for $\mathrm{Na} \alpha-$ dox $1 \mathrm{P}<0.004)$.

The key hormone for responses during pathogen infection salicylic acid (SA) was not significantly affected by the feeding of $M$. sexta but by the expression of $\alpha$-DOX in the transformed lines (Additional file 4D, E). SA tended to be reduced in all $\alpha$-dox silenced plants with and without herbivory by $M$. sexta, however, this trend was only significant for the comparison between WT plants inoculated with VIGSev and VIGS $\alpha$-dox2.

Feeding by $M$. sexta significantly increased ABA levels (Figure 5B). Constitutive and M. sexta-induced levels of ABA are significantly enhanced in plants silenced for both Na $\alpha$-dox genes (Fisher's PLSD VIGS $\alpha$-dox2 in 


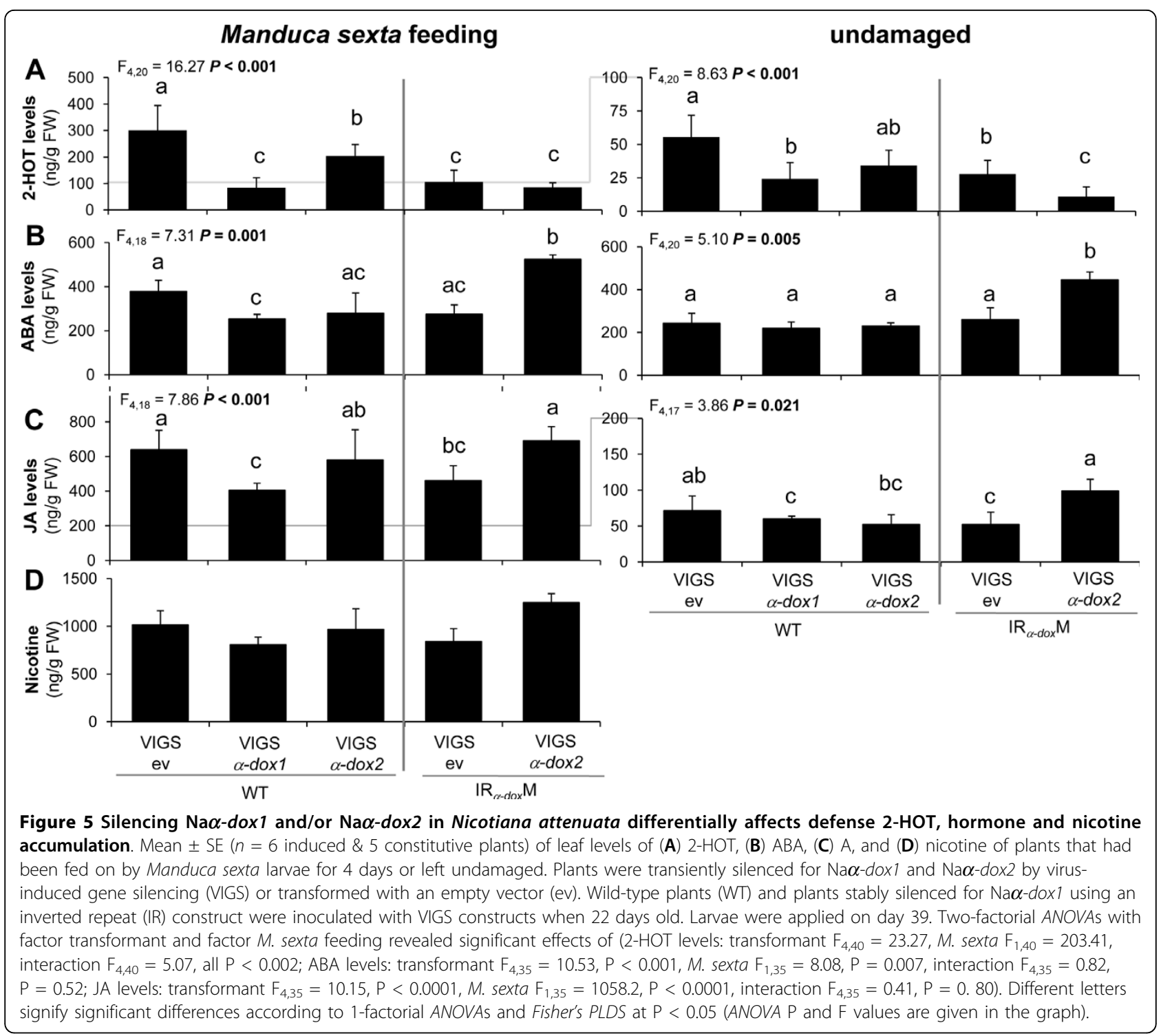

$\mathrm{IR}_{\alpha-\text { dox }} \mathrm{M}$ vs. all other transformants $\left.\mathrm{P}<0.001\right)$. Silencing Na $\alpha$-dox 1 decreases $M$. sexta-induced ABA levels, as ABA levels in VIGS $\alpha$-dox 1 plants were significantly lower compared to VIGSev WT plants and the same tendency was observed in VIGSev in $\mathrm{IR}_{\alpha \text {-dox }} \mathrm{M}$ plants (Fisher's PLSD VIGSev in WT vs. VIGSev in $\mathrm{IR}_{\alpha \text {-dox }} \mathrm{M}$ $\mathrm{P}=0.06$ ). As expected, JA levels were strongly increased in response to $M$. sexta feeding (Figure $5 \mathrm{C}$ ). The levels of induced JA were lower in plants transiently and stably silenced only for Na $\alpha$-dox 1 compared to VIGSev WT (Fisher's PLSD VIGSev vs. VIGS $\alpha$-dox1 P $=0.0007$, VIGSev in WT vs. VIGSev in $\left.\mathrm{IR}_{\alpha-d o x} \mathrm{M} P=0.007\right)$, but not in those silenced for $\alpha$-dox2 (Fisher's PLSD VIGSev vs. VIGS $\alpha$-dox2 P = 0.32). Plants silenced for both Na $\alpha$-dox genes had higher levels of JA compared to plants silenced for $\mathrm{Na} \alpha$-dox 1 alone, which was already apparent in constitutive JA levels (Fisher's PLSD VIGS $\alpha$ dox 2 in $\mathrm{IR}_{\alpha \text {-dox }} \mathrm{M}$ vs. other transformants $\mathrm{P}<0.03$ except vs. VIGSev WT P $=0.70$ ) and did not result from an increased induction after $M$. sexta feeding. Induced nicotine accumulation in VIGS plants corresponded well with the observed JA induction pattern and was lower in the plants only silenced for $\mathrm{Na} \alpha-d o x 1$, but not in those silenced for $\mathrm{Na} \alpha$-dox2 (Figure 5D). However, the data with heterogeneous variances were not normally distributed and no transformation for the nicotine data allowed for parametric statistical analysis; the Mann-Whitney $U$-test only detected a significant difference between plants silenced for $\mathrm{Na} \alpha-d o x 1$ and those silenced for both $\mathrm{Na} \alpha$-dox genes (VIGS $\alpha$-dox 1 WT vs. VIGS $\alpha$-dox 2 in $\mathrm{IR}_{\alpha-\text { dox }} \mathrm{M} \mathrm{P}=0.01$ ) because nicotine levels were highest in the latter plants. 
These results indicate that the effects of individual and simultaneous silencing of the two Na $\alpha$-dox genes exhibit opposing effects likely resulting from different causes. Whereas stable and transient silencing of the $\mathrm{Na} \alpha$-dox 1 gene weakens the herbivore-induced plant response with respect to JA and ABA as well as secondary metabolite production, the silencing of both $\mathrm{Na} \alpha$-dox genes, which stunts growth, results in higher constitutive levels of diverse stress-related traits, some of which are likely to also be elicited by herbivore attack.

\section{Discussion}

The function of the $\alpha$-DOX pathway, which is widespread in the plant kingdom, is poorly understood. Here we identify the $\mathrm{Na} \alpha-d o x 2$ gene in $N$. attenuata and demonstrate that $\alpha$-oxidation of fatty acids plays an important role during its development and that $\mathrm{Na} \alpha$ dox 1 and $\mathrm{Na} \alpha$-dox 2 differ in their transcriptional regulation. Both genes are transcriptionally up-regulated during leaf maturation and senescence and silencing both genes results in retarded growth of $N$. attenuata plants. However, the distinct expression patterns of the two $\mathrm{Na} \alpha$-dox genes indicate a more important role of the $\mathrm{Na} \alpha$-dox 2 gene during plant development, especially in root and flower development and seedling maturation. The strong transcriptional regulation of $\mathrm{Na} \alpha-d o x 1$ in response to herbivory and the reduced induction of JA when $\mathrm{Na} \alpha$-dox 1 is silenced suggest a role in plant defense responses, whereas $\mathrm{Na} \alpha$-dox 2 shows no transcriptional regulation in response to herbivory and its silencing did not change the elicitation of JA. We infer from the different patterns of transcriptional regulation that the two $\mathrm{Na} \alpha$-dox genes contribute differently to functions in primary and secondary plant metabolism in N. attenuata.

\section{Functional diversification of plant $\alpha$-dox genes}

We identified the Na $\alpha$-dox 2 gene and obtained a 2290 bp cDNA sequence from a full transcriptome data base of $N$. attenuata. The Na $\alpha$-dox2 likely corresponds to an orthologue of Arabidopsis and tomato $\alpha$-dox genes with which they share $89 \%$ and $79 \%$ sequence homology, whereas sequence homology between $\mathrm{Na} \alpha$-dox 1 and $\mathrm{Na} \alpha$-dox 2 is $69 \%$. The duplication of $\alpha$-dox genes likely occurred early in the evolution of eudicots before the split of Solanaceae and Brassicaceae, since the orthologues between species contain significantly more homologies than do the paralogues within species.

Stable transformation of $N$. attenuata plants with a silencing construct for $\mathrm{Na} \alpha$-dox 1 resulted in two distinct growth phenotypes. Whereas growth and development of $\mathrm{IR}_{\alpha \text {-dox }} \mathrm{M}$ lines with a ca. $40 \%$ reduction of $\alpha$-DOX activity were indistinguishable from those of WT plants, about $60 \%$ of the generated lines with low or undetectable $\alpha$-DOX activity were highly stunted $\left(\mathrm{IR}_{\alpha-}\right.$ $\left.d_{o x} S\right)$. Stable transformations were performed before the $\alpha$-dox 2 paralogues were known and gene specific real time PCR revealed that these two phenotypes correlated with a dissimilar silencing of $\mathrm{Na} \alpha-d o x 1$ and $\mathrm{Na} \alpha$-dox 2 in the respective lines. Whereas $\operatorname{IR}_{\alpha-d o x} \mathrm{M}$ plants are reduced in Na $\alpha$-dox 1 transcript accumulation by $40 \%$, they are not significantly altered in $\mathrm{Na} \alpha$-dox 2 transcript accumulation. $\mathrm{IR}_{\alpha-d o x} \mathrm{~S}$ lines, on the other hand, are reduced by $85 \%$ in $\mathrm{Na} \alpha-d o x 1$ and $70 \%$ in $\mathrm{Na} \alpha-d o x 2$ transcript accumulation. The $\mathrm{Na} \alpha-d o x 1$ silencing construct shares $72 \%$ sequence similarity with the $\mathrm{Na} \alpha$ dox 2 gene and contains a 24 nucleotide match with only one mismatch, which was probably sufficient for the cosilencing of both $\mathrm{Na} \alpha$-dox genes in the majority of transformed lines. The stunted growth could either result from a concomitant silencing of both $\mathrm{Na} \alpha-d o x$ genes or the loss of the $\mathrm{Na} \alpha-d o x 2$ function alone. The latter hypothesis is consistent with the stunted growth phenotype of feebly [26] and divaricata mutants [32] in tomato, which result from insertional mutagenesis at the locus of the Sl $\alpha$-dox 2 homologue. However, Arabidopsis mutants of At $\alpha$-dox 2 do not show any developmental abnormalities, indicating that the developmental role of $\alpha$-dox 2 may be restricted to a specific clade e.g. Solanaceae. Alternatively, $\alpha$-dox functions in $N$. attenuata may also be distinct from those in tomato, and the stunted growth of in $\mathrm{IR}_{\alpha \text {-dox }} \mathrm{S}$ lines is the consequence of an almost complete loss of total $\alpha$-DOX activity due to a silencing of both Na $\alpha$-dox genes.

\section{Silencing both $\mathrm{Na} \alpha$-dox genes is required to stunt plant growth}

By using VIGS-constructs specific for $\mathrm{Na} \alpha$-dox 1 and $\mathrm{Na} \alpha-d o x 2$ we recognized that silencing $\mathrm{Na} \alpha-d o x 1$ and $\mathrm{Na} \alpha$-dox 2 individually did not affect growth, but silencing both genes concomitantly decreased plant growth. This finding is consistent with the hypothesis that the stunted growth phenotype results from a reduction in total $\alpha$-DOX activity and indicates that the $\alpha$-oxidation activity of the enzymes of both genes can compensate for the loss of function of the other. With in vitro assays, Bannenberg et al. [28] demonstrated that tomato Sl $\alpha$-dox 1 and Sl $\alpha$-dox 2 have a similar range of fatty acid substrates and activities. In $N$. attenuata both Na $\alpha$-dox genes can function in plant development, whereas in tomato the loss of Sl $\alpha$-dox 2 function is sufficient to stunt growth and in Arabidopsis the loss of both At $\alpha$ dox genes does not affect growth [28]. The normal growth of $N$. attenuata plants silenced for $\mathrm{Na} \alpha$-dox 2 by VIGS may alternatively be explained by a lower silencing efficiency compared to the Sl $\alpha$-dox 2 tomato insertional mutant, however this seems unlikely given that VIGS reduced $\mathrm{Na} \alpha$-dox 2 transcripts by $80 \%$ and silencing by 
stable transformation only reduced transcript levels by $70 \%$ in the dwarf $\mathrm{IR}_{\alpha-d o x} \mathrm{~S}$ plants.

That the stunted growth in stably transformed $\mathrm{IR}_{\alpha-}$ ${ }_{d o x} \mathrm{~S}$ lines is more pronounced than in the VIGS plants co-silenced for $\mathrm{Na} \alpha$-dox 1 and $\mathrm{Na} \alpha-d o x 2$ is probably due to greater effects of silencing a gene early than later in development. The growth of $\mathrm{IR}_{\alpha-d o x} \mathrm{~S}$ plants was already delayed two weeks after germination. Though we did not observe obvious growth differences between WT and $\mathrm{IR}_{\alpha-\text {-dox }} \mathrm{S}$ seedlings, the importance of $\alpha$-DOX activity during seedling maturation in $N$. attenuata is supported by highly increased levels of anthocyanins in $\mathrm{IR}_{\alpha-}$ ${ }_{d o x} S$ seedlings. Increased levels of anthocyanins are also described for tomato mutants lacking Sl $\alpha$-dox2 function [28]. The importance of $\alpha$-oxidation of fatty acids during germination processes is also suggested by the increased transcript accumulation and enzymatic activity of a Ps $\alpha$-dox during the first days of germinating pea plants [23]. Increased levels of anthocyanins and ABA are often associated with oxidative stress [33] and levels of ABA are also increased in $\mathrm{IR}_{\alpha-d o x} \mathrm{~S}$ plants. Additionally, constitutive levels of the phytohormones JA and SA are increased in $\mathrm{IR}_{\alpha-d o x} \mathrm{~S}$ plants. Thus, strong silencing of both $\mathrm{Na} \alpha$-dox genes in $\mathrm{IR}_{\alpha \text {-dox }} \mathrm{S}$ plants could have led to severe physiological stress resulting in increased levels of several stress-related metabolites such as several phytohormones. Alternatively, higher levels of constitutive phytohormones could be a consequence of less cellular expansion in these lines, rather than specific effects on specific metabolic pathways. None of these changes were observed in $\mathrm{IR}_{\alpha-d o x} \mathrm{M}$ plants indicating that a moderate silencing of $\mathrm{Na} \alpha$-dox 1 does not result in comparable physiological stress during development.

\section{Both Na $\alpha$-dox genes have overlapping but distinct roles in plant development}

Transcript accumulation of $\mathrm{Na} \alpha-d o x 1$ and $\mathrm{Na} \alpha-d o x 2$ in various tissues of developing $N$. attenuata plants were determined to characterize their endogenous roles. In roots, transcript accumulation of $\mathrm{Na} \alpha-d o x 2$ is very high, whereas it is very low for $\mathrm{Na} \alpha-\operatorname{dox} 1$, which is consistent with previous studies in $N$. attenuata [5] that found no $\mathrm{Na} \alpha$-dox 1 expression in roots. The root specific expression of $\mathrm{Na} \alpha$-dox 2 distinguishes $N$. attenuata from pea, Arabidopsis and tomato, in which $\alpha$-dox 1 is expressed in roots but not $\alpha$-dox2. Bannenberg et al. [28] found no detectable At $\alpha$-dox 2 transcripts in roots of Arabidopsis and only very low levels of Sl $\alpha$-dox 2 transcripts but high levels of one $\operatorname{Sl} \alpha$-dox 1 isoform in tomato roots. In Arabidopsis, the At $\alpha-d o x 1$ promoter is exclusively active in roots whereas the At $\alpha$-dox 2 promoter is exclusively active in shoots $[19,28,34]$. In pea seedlings, Ps $\alpha$-dox is almost exclusively expressed in roots and this gene shares a greater degree of amino acid sequence similarity with Nt $\alpha$-dox 1 and At $\alpha$-dox 1 than with At $\alpha$ dox2 [23]. In this regard, the stunted phenotype of $\mathrm{IR}_{\alpha-}$ ${ }_{d o x} \mathrm{~S}$ lines could also result from silencing $\mathrm{Na} \alpha-d o x 2$, which may have interfered with root development causing the observed growth and stress responses. $\mathrm{IR}_{\alpha-\text { dox }} \mathrm{S}$ lines are characterized by a small main root and very few lateral roots; to determine whether this is a cause or a consequence of the dwarf growth, grafting experiments would be required. However, the loss of function of $\mathrm{Na} \alpha-d o x 2$ in root development can only contribute to but not cause the dwarf phenotype, as the silencing of both $\mathrm{Na} \alpha$-dox genes is required to stunt growth. In $N$. attenuata, the transcripts of both $\mathrm{Na} \alpha$-dox genes accumulate during leaf maturation and natural senescence with levels of $\mathrm{Na} \alpha$-dox 1 exceeding those of $\mathrm{Na} \alpha$-dox 2 . This is in contrast to tomato and Arabidopsis, in which At $\alpha$-dox 2 and Sl $\alpha$-dox 2 transcripts only accumulate in response to the artificial senescence stimulus of leaf detachment $[27,28]$, whereas neither $\alpha$-dox 1 nor $\alpha$-dox 2 genes accumulate in leaves attached to plants. Moreover, in contrast to $N$. attenuata, in which the levels of $\mathrm{Na} \alpha$ dox 1 transcripts exceed those of $\mathrm{Na} \alpha-d o x 2$, in Arabidopsis and tomato At $\alpha$-dox 1 and Sl $\alpha$-dox 1 transcripts are barely detected in leaves and showed only an extremely weak response to leaf detachment. However, the results in $N$. attenuata are in line with the increasing expression of $\mathrm{Na} \alpha$-dox 1 gene during natural leaf senescence in $N$. tabacum [24]. We propose that in Nicotiana species $\alpha$-dox genes may be involved in the regulation of senescence, whereas in Arabidopsis and tomato increasing oxidative stress in the dying tissue of a detached leaf may be at work. The loss of function of both $\mathrm{Na} \alpha-d o x$ genes during leaf maturation may be involved in the dwarf phenotype of the $\mathrm{IR}_{\alpha-d o x} \mathrm{~S}$ lines as the overlapping transcriptional regulation of $\mathrm{Na} \alpha-d o x 1$ and $\mathrm{Na} \alpha-d o x 2$ in leaves corresponds well to the observation that silencing of both genes is necessary to stunt growth.

During flower maturation in $N$. attenuata, only $\mathrm{Na} \alpha$ dox2 transcripts accumulate, so that they are twofold higher in mature flowers than in flower buds. Consistent with Hermsmeier et al. [5], who found no transcripts of Na $\alpha$-dox 1 in flowers of $N$. attenuata, we found very low transcript abundance of $\mathrm{Na} \alpha$-dox 1 in flowers. However, in Arabidopsis, promoters of both At $\alpha$-dox genes are active in anthers and the At $\alpha$-dox 2 promoter was additionally shown to be active in the ovaries and siliques $[19,28]$. In distylous species of Turnera, a homologue of $\alpha$-dox 1 is specifically expressed in short-styled plants [35]. Interestingly, long chain aldehydes, similar to those generated in vitro by the activity of $\alpha$-DOX proteins, are emitted by many flowers [36]; however, the role of $\alpha$-dox genes during flower maturation remains unknown and is likely not involved in causing the stunted phenotype. 
Only Na $\alpha$-dox 1 is involved in responses to herbivory The similar regulation of the two $\mathrm{Na} \alpha-d o x$ genes in $N$. attenuata leaves is restricted to developmental regulation, however in contrast to $\mathrm{Na} \alpha$-dox 1 , the $\mathrm{Na} \alpha-d o x 2$ gene does not respond to wounding or the elicitors in the OS of $M$. sexta larvae. In agreement with its upregulation during herbivory, $\mathrm{Na} \alpha$-dox 1 contributed most to the herbivore-inducible 2-HOT level. Silencing of $\mathrm{Na} \alpha$-dox 1 by VIGS or stable transformation reduced herbivore-inducible 2 -HOT levels by $60 \%$ while silencing only $\mathrm{Na} \alpha-d o x 2$ had only a marginal effect. Accordingly, we found no effect of silencing Na $\alpha$-dox 2 by VIGS on induced responses to $M$. sexta herbivory, whereas stable and transient silencing of $\mathrm{Na} \alpha-d o x 1$ decreased the M. sexta-induced levels of JA by $30 \%$ and tended to decrease induced levels of the direct defense metabolite, nicotine. VIGS of Na $\alpha$-dox 1 also reduced $M$. sextainduced ABA levels by $25 \%$. Thus, $\mathrm{Na} \alpha$-dox 1 plays an important role in the regulation of the plant's response to herbivory, whereas silencing $\mathrm{Na} \alpha-d o x 2$ did not affect induced phytohormone levels. VIGS of both Na $\alpha$-dox genes concomitantly only increased constitutive levels of $\mathrm{JA}$ and ABA and strongly decreased constitutive levels of 2-HOT which is consistent with the phytohormone phenotype in stably transformed plants co-silenced for $\mathrm{Na} \alpha$-dox 1 and $\mathrm{Na} \alpha-d o x 2$. Because plants silenced for both $\mathrm{Na} \alpha-d o x$ genes display the loss of function in development and in the plant's response to herbivory, the specific role of $\mathrm{Na} \alpha$-dox 1 during herbivory should be further investigated using $\mathrm{IR}_{\alpha-d o x} \mathrm{M}$ plants and plants silenced by VIGS for Na $\alpha$-dox1. A kinetic analysis of JA induction in the $\mathrm{Na} \alpha$-dox 1 silenced plants may reveal potential feedback regulation by 2 -HOT, as we show here that $\mathrm{Na} \alpha$-dox 1 gene expression is mediated by but also affects JA signaling.

\section{Conclusions}

In this study we identified the $\alpha$-dox 2 isoform in $N$. attenuata and revealed that both $\mathrm{Na} \alpha-d o x$ genes function in development but that only $\mathrm{Na} \alpha-d o x 1$ functions in anti-herbivore defense. The conditions that regulate the two $\alpha$-dox isoforms and the consequences of their loss of function in $N$. attenuata and other plants share commonalities and clear differences supporting quite divergent functions in different plant species. Interestingly, phylogenetic relation is a poor predictor of the regulation of $\alpha$-dox genes in different plant species (see Additional file 5 for a summary of the putative functions in a phylogenetic context), but transcriptional regulation is suggested to determine function of $\alpha$-dox genes, because the enzymatic activity is largely similar for the $\alpha$-dox paralogues within species as well as for the orthologes among species. This suggests that the $\alpha$-DOX pathway is a relatively plastic metabolic route in plant primary metabolism which became repeatedly co-opted for specialized functions associated with adaptation to environmental stress. A better mechanistic understanding of the role of $\alpha$-dox genes during plant development and stress responses will help to explain the differences and seemingly convergent similarities of $\alpha$-dox regulation in different plant species.

\section{Methods}

\section{Plant material \& transformation}

We used inbred lines of Nicotiana attenuata Torr. ex Watson derived from field-collected seeds (Baldwin, 1998) for transformation and all experiments. Seed germination and Agrobacterium tumefaciens (strain LBA 4404)-mediated transformation procedure are described in Krügel et al. [37]. All plants were grown in the glasshouse in $1 \mathrm{~L}$ pots at $26-28^{\circ} \mathrm{C}$ under $16 \mathrm{~h}$ of light supplied by Philips Sun-T Agro 400- or 600-W sodium lights.

To silence the expression of the $N$. attenuata $\alpha$-dox 1 gene [GenBank AF229926], a 458 bp fragment [5] was cloned twice in an inverted-repeat orientation into a pRESC5 transformation vector. T1 plants were screened for resistance to hygromycin (hygromycin phosphotransferase II gene from pCAMBIA-1301 [GenBank AF234297] contained in the pRESC5 vector). Homozygosity was determined by resistance screening of the T2 plants. Flow cytometric analysis described by Bubner et al. [38] revealed that all the lines were diploid. Each transformed line harbored only a single insertion, as determined by Southern blot analysis (Additional file 3C) performed on EcoRV-digested genomic DNA with a probe specific for the selective marker gene hygromycin phosphotransferaseII (plasmids used for transformation were included as a positive control and additionally digested with XhoI). For the experiments we used two $\mathrm{IR}_{\alpha-d o x} \mathrm{~S}$ lines (line 1: A03-77; line 2: A03-137) with stunted and two $\mathrm{IR}_{\alpha-d o x} \mathrm{M}$ lines with normal growth (line 1: A03-282; line 2: A03-75), whereas $\mathrm{IR}_{\alpha-\text { dox }} \mathrm{M}$ line 2 was used in the VIGS experiment.

For virus induced gene silencing, PCR amplified fragments of $\mathrm{Na} \alpha-d o x 1$ and $\mathrm{Na} \alpha$-dox2 (each fragment was $187 \mathrm{bp}$ long, for specific sequence see Additional file 1) were digested with BamHI and SalI and each cloned into the PTV00 vector digested with the same restriction enzymes. The pTV00 vector is a $5.5-\mathrm{kb}$ plasmid with an origin of replication for Escherichia coli and A. tumefaciens and a gene for kanamycin resistance (Ratcliff et al., 2001). Early rosette-stage plants (3 weeks old) were pressure-injected with $A$. tumefaciens (strain GV3101) harboring either the pTV $\alpha$-dox $1, \mathrm{pTV} \alpha$-dox 2 or the empty vector (ev) control pTV00. A phytoene desaturase-silencing construct ( $\mathrm{pTV} p d s$ ) that causes photobleaching was used as a visible positive control for the 
VIGS process in all experiments. Inoculation and constructs are described in Saedler and Baldwin [39]. When the leaves of phytoene desaturase-silenced plants began to bleach (17 days after inoculation) leaves of $\alpha$-doxsilenced and ev-inoculated plants were used.

\section{Plant treatments}

Experiments with stable transformants were performed with $\mathrm{T}_{3}$ generation $\mathrm{IR}_{\alpha-\text { dox }}$ plants. Analysis of induced responses (phytohormones, $\alpha$-DOX activity, and transcriptional changes) was performed with four to five week-old (rosette-stage) plants and treatments were randomly assigned. To mimic herbivory, the first fully expanded source leaf was wounded four times with a fabric pattern wheel with a row of puncture wounds on each side of the midrib and each row received $5 \mu \mathrm{L}$ of a 1:1 v/v dilution of the oral secretions of $M$. sexta. At specified time points, leaves were excised, immediately frozen in liquid nitrogen, and stored at $-80^{\circ} \mathrm{C}$ until use.

$\mathrm{Na} \alpha-d o x 1$ and $\mathrm{Na} \alpha$-dox 2 constitutive transcript abundances were measured in different tissue parts from WT $N$. attenuata plants. The entire young rosette-stage plant was harvested 30 days after germination. Roots, green or senescing yellow rosette leaves, stem leaves (S5-S8) and flower buds were harvested from 54-day old plants. Complete flowers were harvested 62 days after germination.

Plants that had been subject to virus-induced gene silencing were measured for rosette diameter 17 days after inoculation (at which time there were no differences in plant size) and for stalk elongation 38 days after inoculation. Half of the plants received a M. sexta neonate on the transition leaf after inoculation and these larvae were allowed to feed for 4 days. On day 4 , leaves on which neonates were feeding were harvested for metabolite measurements. Eggs of $M$. sexta were from North Carolina State University (Raleigh, NC, USA) and maintained in a growth chamber (Snijders Scientific, Tilburg, Netherlands) at $26^{\circ} \mathrm{C} 16 \mathrm{~h}$ light, $24^{\circ} \mathrm{C}$ $8 \mathrm{~h}$ darkness until the larvae hatched.

\section{Identification of the Na $\alpha$-dox 2 gene}

Primers were designed according to a consensus sequence of $\alpha$-dox 2 isoforms in S. lycopersicum and $A$. thaliana which have a low sequence identity to the Na $\alpha$-dox 1 gene. The resulting PCR amplicon was cloned into pGEM-T Easy plasmid (Promega), transformed into Escherichia coli competent cells (JM109) and subsequently sequenced on a Genetic Analyzer 3100 (Applied Biosystems, Darmstadt, Germany) from 3 independent clones. The resulting 355 bp gene fragment of the $\mathrm{Na} \alpha-$ dox2 was compared to a data base of the complete transcriptome of $N$. attenuata (VERTIS Biotechnologie AG,
Germany) and a 2290 bp cDNA sequence of the Na $\alpha$ dox2 gene was identified.

\section{Analysis of transcript expression by quantitative real time PCR}

Total RNA was extracted with trizol following the TIGR protocol available under http://www.jcvi.org/potato/sol_ma_protocols.shtml and cDNA was synthesized from 150 ng RNA using MultiScribe ${ }^{\text {Tw }}$ reverse transcriptase (Applied Biosystems, Darmstadt, Germany). Quantitative real time PCR (ABI PRISM ${ }^{\mathrm{Tw}} 7000$; Applied Biosystems) was conducted using the quantitative $\mathrm{PCR}^{\mathrm{ma}}$ core reagent kit (Eurogentec, Seraing, Belgium) and gene specific primers for SYBR Green-based quantitative PCR (Na $\alpha$ dox 1 forward primer: GTGTTGCTAGGTACAATGAA TTTC; Na $\alpha$-dox 1 reverse primer: CAACCATCAGATCCAATTCTTCT; Na $\alpha$-dox 2 forward primer: GTTCCACGGTACAACGAGTTCA; Na $\alpha$-dox 2 reverse primer: AACTTGGAGATCTAGCTTCTCA). PCR conditions were used according to the manufacturer's recommendations. For each treatment/line combination cDNA of 3 biological replicates were repeated with 3 technical replicates. Relative expression levels were calculated according to a linear standard curve of the threshold cycle number regressed against the log concentration of a dilution series of cDNA standards that were analyzed on each 96-well plate. The expression of actin [GenBank EU273278.1] was used as an internal reference to normalize cDNA concentrations.

\section{Analysis of $\alpha$-DOX activity}

Leaves (pooled from 5 plants) were crushed in liquid nitrogen and $250 \mu \mathrm{L}$ extraction buffer $(0.1 \mathrm{M}$ Tris- $\mathrm{HCl}$ $\mathrm{pH}$ 7.5) were used for every $100 \mathrm{mg}$ tissue. Leaf tissues were subsequently completely homogenized by being vortexed at $4^{\circ} \mathrm{C}$ for $45 \mathrm{~min}$. After centrifugation, protein concentration of the resulting supernatants was measured using a Bio-Rad protein assay kit with bovine serum albumin (BSA) as a standard. The $\alpha$-DOX activity was assayed indirectly by measuring HDT formation in response to $\alpha$-linolenic acid (C18:3) incubation. The reaction mixture consisted of $200 \mu \mathrm{M} \mathrm{C} 18: 3$ incubated with $200 \mu \mathrm{L}$ of protein solution and Tris- $\mathrm{HCl}$ buffer $(0.1$ $\mathrm{M}, \mathrm{pH}$ 7.5) in a total volume of $500 \mu \mathrm{L}$. $\alpha$-DOX products and the residual C18:3 were extracted with ethyl acetate; HDT was quantified by reverse-phase HPLC-UV after 2,4-dinitrophenylhydrazine derivatization, following the established procedure described in Kohlmann et al. [40].

\section{Analysis of secondary metabolites and phytohormones and 2-HOT}

Nicotine was analyzed by HPLC as described previously (Keinanen et al., 2001) with the following modification 
of the extraction procedure: approximately $100 \mathrm{mg}$ of frozen tissue was homogenized in $1 \mathrm{~mL}$ of extraction buffer using the FastPrep extraction system (Savant Instruments, NY, USA). Samples were homogenized in FastPrep tubes containing $900 \mathrm{mg}$ of lysing matrix (BIO 101) by shaking at $6.0 \mathrm{~m} / \mathrm{s}$ for $45 \mathrm{~s}$.

To analyze phytohormones and 2-hydroxy-octadecatrienoic acid (2-HOT), $200 \mathrm{mg}$ leaf samples were extracted with ethyl acetate containing $40 \mathrm{ng} / \mathrm{ml} \mathrm{D}_{6}$ ABA, $\mathrm{D}_{4}$-SA and JA- ${ }^{13} \mathrm{C}_{6}$-Ile and $200 \mathrm{ng} / \mathrm{ml}$ of $\mathrm{D}_{2}$-JA according to $\mathrm{Wu}$ et al. [41]. A $10 \mu \mathrm{l}$ aliquot of the resulting extracts was analyzed by reverse-phase HPLC coupled to tandem mass spectrometry (HPLC/ESI-MS/ MS). Phytohormones were separated from extracts at a flow rate of $100 \mu \mathrm{L} \mathrm{min}{ }^{-1}$ on a Pursuit C8 column $(3 \mu \mathrm{m}, 150 \times 2 \mathrm{~mm}$; Varian) using a binary solvent system (A: $0.05 \% v / v$ formic acid in deionized water; B: $0.05 \% v / v$ formic acid in methanol) in gradient mode. Multiple reaction monitoring (MRM) was conducted on a 1200 L MS/MS system (Varian, Palo Alto, CA, USA), operated in negative ionization mode. Parent-ion/daughter-ion selections and collision energies were set as follows: $213 / 59$ ( $\mathrm{D}_{2}$-dihydro-JA, 12V), 209/59 (JA, 12V), 328/136 ( ${ }^{13} \mathrm{C}_{6}$-JA-Ile, 19V), 322/130 (JA-Ile, 19V), 269/ 159 (D D $_{6}$ ABA, 9V), 263/153 (ABA, 9V), 141/97 (D4-SA, 15V), 137/93 (SA, 15V) and 293/191 (2-HOT). The area beneath the MRM product ion peak was determined for each analyte and internal standard (IS). The quantity of the analyte was calculated according to the formula: analyte product ion peak area $\times$ (IS concentration/IS product ion peak area). A calibration curve obtained by the extraction and analysis of a pool leaf sample spiked with increasing of amounts 2-HOT (Larodan Fine Chemicals, Sweden) was used for 2-HOT quantification.

For anthocyanin measurements 11, 15 and 19 days old seedlings of WT and two $\mathrm{IR}_{\alpha-d o x} \mathrm{~S}$ lines were extracted according to Rabino and Mancinelli [42]. Approximately 30 seedlings $(100 \mathrm{mg})$ were harvested, immediately frozen in liquid nitrogen and stored at $-80^{\circ} \mathrm{C}$ for at least 20 minutes. After grinding $200 \mu \mathrm{l}$ of $1 \% \mathrm{HCl} /$ methanol solution were added and incubated overnight $(15 \mathrm{~h})$ at room temperature with constant vortexing. After centrifugation (5000 g, $5 \mathrm{~min}$ ) the absorbance of the supernatant and a dilution series of cyanidin-5-O-glucoside at $530 \mathrm{~nm}$ and $657 \mathrm{~nm}$ was determined. The amount of anthocyanin was calculated according to the absorbance difference $(530 \mathrm{~nm}-657 \mathrm{~nm})$.

\section{Statistics}

Data were analyzed using the statistic program StatView ${ }^{\circ}$ 5.0 (SAS Institute Inc., Cary, NC, USA). All data were checked graphically for normal distribution and for variance homogeneity using the $F$-test and transformed if required to meet these assumptions. The general level of significance applied was $\alpha=0.05$.

The effects of Na $\alpha$-dox silencing on $\alpha$-DOX activity, $\mathrm{Na} \alpha-d o x 1$ and $\mathrm{Na} \alpha-d o x 2$ transcript accumulation, and levels of ABA and JA in leaves were determined by comparing transformed lines against the WT $N$. attenuata by unpaired $t$-tests. Data for transcript accumulation were cube-root transformed for normality and variance homogeneity. Because no transformation was found to meet the requirements for parametric statistical analyses, levels of anthocyanins were compared between WT and $\mathrm{IR}_{\alpha \text {-dox }} \mathrm{S}$ lines by Mann-Whitney $U$-tests.

The tissue-specific expression was compared between the different tissues by individual 1-factorial ANOVAs followed by Fisher's PLSD for Na $\alpha$-dox 1 and the Na $\alpha$ dox 2 gene. The transcript accumulation of $\mathrm{Na} \alpha-d o x 1$ and $\mathrm{Na} \alpha-d o x 2$ in each tissue was compared by unpaired $t$-tests. Data were cube root-transformed for normality and variance homogeneity.

The growth parameter and transcript accumulation of $\mathrm{Na} \alpha-d o x 1$ and $\mathrm{Na} \alpha-d o x 2$ for the plants silenced by VIGS were compared for statistically significant differences between transformants by 1 -factorial ANOVA followed by Fisher's PLSD for individual comparisons. Growth data were box cox-transformed ( $\lambda=1$ for rosette diameters and $\lambda=2$ for stem length) and transcript data were cube root-transformed for normality and variance homogeneity. The leaf levels of phytohormones of control and $M$. sexta induced VIGS plants were compared for statistically significant differences by 2 -factorial $A N O V A$ with the factors induction (2 groups: control, $M$. sexta feeding) and silencing (5 groups: VIGSev WT, VIGS $\alpha$-dox 1 WT, VIGS $\alpha$-dox 2 WT, VIGSev in $\operatorname{IR}_{\alpha-d o x} \mathrm{M}$, VIGS $\alpha$-dox 2 in $\mathrm{IR}_{\alpha-d o x} \mathrm{M}$ ) followed by Fisher's PLSD. Additionally, levels of JA, SA and ABA were analyzed by 1-factorial ANOVAs for constitutive and $M$. sexta induced plants separately. Data for JA and SA were log-transformed and data for ABA were cube root-transformed for normality and variance homogeneity. Because no transformation was found to meet parametric assumptions, levels of nicotine in VIGS plants were compared for significant differences between transformants by Mann-Whitney $U$-tests.

\section{Additional material}

Additional file 1: Alignment of Na $\alpha$-dox 1 and Na $\alpha$-dox2 cDNA Sequences were aligned in Bioedit using the ClustalW algorithm. Regions used to design the inverted repeat silencing construct: The region of the $\mathrm{Na} \alpha$-dox 1 gene used for the stable silencing construct is displayed in purple and shares a match of 24 nucleotides with the Na $\alpha$-dox2 gene (containing one mismatch). The virus-induced gene silencing constructs are highlighted in blue for $\mathrm{Na} \alpha-d o \times 1$ and in red for $\mathrm{Na} \alpha$-dox2. 
Additional file 2: Phylogenetic relationship based on deduced amino acids sequences of plant $\alpha$-DOXs. The consensus neighborjoining tree was constructed after 5000 iterations with the MEGA 3.1 software after sequence alignment using the ClustalW algorithm embedded into Bioedit. The GenBank accession numbers of the sequences are displayed onto the tree for the following plant species: Arabidopsis thaliana (At), Capsicum annuum (Ca), Cicer arietinum (Car), Medicago truncatula (Mt), Nicotiana attenuata (Na), Nicotiana tabacum (Nt), Oryza sativa (Os), Physcomistrella patens (Pp), Pisum sativum (Ps), Populus trichocarpa (Pt), Ricinus communis (Rc), Solanum lycopersicon (SI), Turnera subulata (Tsu), Turnera scabra (Tsc), Vitis vinifera (VV).

Additional file 3: Primer specificity, anthocyanin levels, and single insertions. (A) Gel electrophoresis of gene specific PCR products on TAE gel after Ethidium bromide staining. Plasmids $(50 \mathrm{ng})$ containing either the $\mathrm{Na} \alpha$-dox 1 gene (2 left lanes) or a 355 bp fragment of $\mathrm{Na} \alpha$ dox2 (2 right lanes) were amplified with (B) 2 primer pairs, designed to specifically amplify a 144 bp fragment of either $\mathrm{Na} \alpha$-dox 1 (P1) or Na $\alpha$ dox2 (P2). The bands revealed that only primer pair P1 amplified the plasmid containing the $\mathrm{Na} \alpha$-dox 1 gene and only primer pair $\mathrm{P} 2$ amplified the plasmid containing the Nao-dox2 fragment. (B) Anthocyanin levels (mean \pm SE of 3 biological replicates) measured in $100 \mathrm{mg}$ tissue of 11 15, and 19 days old seedlings of WT and $T_{3}$ homozygous Nicotiana attenuata plants transformed with an inverted repeat (IR) construct to silence $\mathrm{Na} \alpha$-dox1 (line 2). (C) Southern analysis of three independently transformed $I R_{\alpha-\text { dox }}$ lines (S1, M1 \& M2) showing single insertion. Genomic DNA $(10 \mu \mathrm{g})$ from individual plants was digested with EcoR1 and blotted onto a nylon membrane. The blot was hybridized with a PCR fragment of the hygromycin phosphotransferase // gene, specific for the selective marker on the T-DNA.

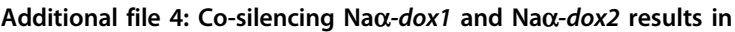
increased constitutive ABA, JA and SA levels. Mean \pm SE $(n=4$ biological replicates) levels of (A) ABA, (B) JA, and (C) SA in untreated rosette leaves of wild-type (WT) Nicotiana attenuata plants transformed with an inverted repeat (IR) construct to silence $\mathrm{Na} \alpha$-dox1. In contrast to lines silenced for $\mathrm{Na} \alpha$-doxionly $\left(\mathbb{R}_{\alpha \text {-dox }} M\right)$, lines that were co-silenced for $\mathrm{Na} \alpha$-dox 1 and Na $\alpha$-dox2 have higher constitutive levels of ABA, JA, and $\mathrm{SA}$. Asterisks signify significant differences between $\mathrm{IR}_{\boldsymbol{\alpha} \text {-dox }}$ and WT plants (unpaired t-test for levels of $\mathrm{ABA}: \mathrm{M} 1 \mathrm{P}=0.65, \mathrm{M} 2 \mathrm{P}=0.30, \mathrm{~S} 1 \mathrm{P}=0.001$, S2 P = 0.03; JA: M1 P = 0.09, M2 P = 0.51, S1 P = 0.001, S2 P = 0.02, SA: $M 1: P=0.43, M 2: P=0.36, S 1 P=0.05, S 2 P=0.002$ ). Leaf SA levels (mean \pm SE of plants that $(\mathbf{D})$ had be fed on by Manduca sexta larvae for 4 days $(n=6)$ or $(\mathbf{E})$ were undamaged $(n=5)$. WT and $\mathbb{R}_{\alpha \text {-dox }} \mathrm{M}$ plants were transiently silenced for $\mathrm{Na} \alpha$-dox 1 and $\mathrm{Na} \alpha$-dox2 by virus-induced gene silencing (VIGS) or transformed with an empty vector (ev). Plants were inoculated with VIGS constructs when 22 days old and Larvae were applied on day 39 . Though SA tended to be reduced in all $\alpha$-dox silenced plants, this was only significant for VIGS Na $\alpha$-dox2 (2-factorial ANOVA: for factor transformant $F_{4,35}=2.73, P=0.04$, for factor $M$. sexta: $F_{1,35}=0.018, P=0.895 ;$ Fischer's PLSD: VIGS ev WT vs. VIGS Na $\alpha$-dox2 $W T P=0.0037$, VIGS ev WT vs. VIGS ev $I_{\alpha \text {-dox }} M P=0.054$ ).

Additional file 5: Table summarizing putative functions of $\alpha$-dox genes in different plant species along the plant phylogenetic tree. The occurrence of $\alpha$-dox genes in different tissues or the regulation by pathogens, herbivores, wounding, or phytohormones it is indicated by "+" (usually a positive regulation except for ethylene in Oryza sativa), whereas the lack of expression is indicated by "-". References are given according to the reference list in the manuscript.

\section{Acknowledgements}

This research was supported by the Max Planck Society. We thank Dr. K. Gase for vector construction, M. Maccani for anthocyanin measurement and both for helping to clone the Na $\alpha$-dox2 gene fragment.

\section{Author details}

'Department of Molecular Ecology, Max-Planck-Institute for Chemical Ecology, Hans-Knöll-Str. 8, Jena 07745, Germany. ${ }^{2}$ Molecular Ecology
Department/Dahlem Centre of Plant Sciences, Institute for Biology/Free University of Berlin, Haderslebener Str. 9, Berlin 12163, Germany.

\section{Authors' contributions}

AS carried out the screening of stably transformed lines, participated in the identification of the Na $\alpha$-dox2 gene, VIGS experiments, the metabolite analyses, performed the statistical analysis and participated in drafting the manuscript. EG performed the transcriptional analysis, participated in the identification of the Na $\alpha$-dox2 gene, VIGS experiments, metabolite analyses, carried out the sequence alignment and participated in drafting the manuscript. ITB conceived the study and participated in its design and coordination and helped to draft the manuscript. All authors read and approved the final manuscript.

Received: 23 March 2010 Accepted: 11 August 2010

Published: 11 August 2010

\section{References}

1. Creelman RA, Mullet JE: Jasmonic acid distribution and action in plants regulation during development and response to biotic and abiotic stress. Proc Natl Acad Sci USA 1995, 92:4114-4119.

2. Blee E: Impact of phyto-oxylipins in plant defense. Trends Plant Sci 2002, 7:315-322.

3. Halitschke R, Baldwin IT: Antisense LOX expression increases herbivore performance by decreasing defense responses and inhibiting growthrelated transcriptional reorganization in Nicotiana attenuata. Plant J 2003, 36:794-807.

4. Creelman RA, Mullet JE: Biosynthesis and action of jasmonates in plants. Annu Rev Plant Physiol Plant Mol Biol 1997, 48:355-381.

5. Hermsmeier D, Schittko U, Baldwin IT: Molecular interactions between the specialist herbivore Manduca sexta (Lepidoptera, Sphingidae) and its natural host Nicotiana attenuata. I. Large-scale changes in the accumulation of growth- and defense-related plant mRNAs. Plant Physiol 2001, 125:683-700.

6. Schittko U, Hermsmeier D, Baldwin IT: Molecular interactions between the specialist herbivore Manduca sexta (Lepidoptera, Sphingidae) and its natural host Nicotiana attenuata. II. Accumulation of plant mRNAs in response to insect-derived cues. Plant Physiol 2001, 125:701-710.

7. Voelckel C, Baldwin IT: Herbivore-induced plant vaccination. Part II. Arraystudies reveal the transience of herbivore-specific transcriptional imprints and a distinct imprint from stress combinations. Plant J 2004, 38:650-663.

8. Voelckel C, Baldwin IT: Generalist and specialist lepidopteran larvae elicit different transcriptional responses in Nicotiana attenuata, which correlate with larval FAC profiles. Ecol Lett 2004, 7:770-775.

9. Sanz A, Moreno JI, Castresana C: PIOX, a new pathogen-induced oxygenase with homology to animal cyclooxygenase. Plant Cell 1998, 10:1523-1537.

10. Hamberg M, Sanz A, Castresana C: $\alpha$-Oxidation of fatty acids in higher plants - identification of a pathogen-inducible oxygenase (PIOX) as an $\alpha$-dioxygenase and biosynthesis of 2-hydroperoxylinolenic acid. J Biol Chem 1999, 274:24503-24513.

11. Hamberg M, Sanz A, Rodriguez MJ, Calvo AP, Castresana C: Activation of the fatty acid alpha-dioxygenase pathway during bacterial infection of tobacco leaves - Formation of oxylipins protecting against cell death. J Biol Chem 2003, 278:51796-51805.

12. Prost I, Dhondt S, Rothe G, Vicente J, Rodriguez MJ, Kift N, Carbonne F, Griffiths G, Esquerre-Tugaye MT, Rosahl S, et al: Evaluation of the antimicrobial activities of plant oxylipins supports their involvement in defense against pathogens. Plant Physiol 2005, 139:1902-1913.

13. Tirajoh A, Aung TST, MCKay AB, Plant AL: Stress-responsive alphadioxygenase expression in tomato roots. J Exp Bot 2005, 56:713-723.

14. Seki M, Narusaka M, Ishida J, Nanjo T, Fujita M, Oono Y, Kamiya A, Nakajima M, Enju A, Sakurai T, et al: Monitoring the expression profiles of 7000 Arabidopsis genes under drought, cold and high-salinity stresses using a full-length cDNA microarray. Plant J 2002, 31:279-292.

15. Koeduka T, Matsui K, Hasegawa M, Akakabe Y, Kajiwara T: Rice fatty acid alpha-dioxygenase is induced by pathogen attack and heavy metal stress: activation through jasmonate signaling. J Plant Physiol 2005, 162:912-920. 
16. Izaguirre MM, Scopel AL, Baldwin IT, Ballare CL: Convergent responses to stress. Solar ultraviolet-B radiation and Manduca sexta herbivory elicit overlapping transcriptional responses in field-grown plants of Nicotiana longiflora. Plant Physiol 2003, 132:1755-1767.

17. Kim Y-C, Yi S-Y, Mang HG, Kim WT, Choi D: Pathogen-induced expression of cylo-oxygenase homologue in hot pepper (Capsicum annuum cv. Pukang). J Exp Bot 2002, 53:383-385.

18. Hamberg M, Ponce de Leon I, Sanz A, Castresana C: Fatty acid alphadioxygenases. Prostaglandins and Other Lipid Mediators 2002, 6869:363-374.

19. Ponce de León I, Sanz A, Hamberg M, Castresana C: Involvement of the Arabidopsis $\alpha$-DOX 1 fatty acid dioxygenase in protection against oxidative stress and cell death. Plant J 2002, 29:61-72.

20. Halitschke R, Schittko U, Pohnert G, Boland W, Baldwin IT: Molecular interactions between the specialist herbivore Manduca sexta (Lepidoptera, Sphingidae) and its natural host Nicotiana attenuata. III. Fatty acid-amino acid conjugates in herbivore oral secretions are necessary and sufficient for herbivore-specific plant responses. Plant Physiol 2001, 125:711-717.

21. Newcomb EH, Stumpf PK: Fatty acid synthesis and oxidation in peanut cotyledons. Phosphorus Metabolism Baltimore: John Hopkins PressMcElroy WD, Glass B 1952, 291-300.

22. Saffert A, Hartmann-Schreier J, Schon A, Schreier P: A dual function alphadioxygenase-peroxidase and $\mathrm{NAD}(+)$ oxidoreductase active enzyme from germinating pea rationalizing alpha-oxidation of fatty acids in plants. Plant Physiol 2000, 123:1545-1552.

23. Meisner AK, Saffert A, Schreier P, Schon A: Fatty acid alpha-dioxygenase from Pisum sativum: Temporal and spatial regulation during germination and plant development. J Plant Physiol 2009, 166(4):333-43.

24. Obregón P, Martin R, Sanz A, Castresana C: Activation of defence-related genes during senescence: a correlationn between gene expression and cellular damage. Plant Mol Biol 2001, 46:67-77.

25. Akakabe Y, Matsui K, Kajiwara T: Enantioselective alpha-hydroperoxylation of long-chain fatty acids with crude enzyme of marine green alga Ulva pertusa. Tetrahedron Lett 1999, 40:1137-1140.

26. van der Biezen EA, Brandwagt BF, vanLeeuwen W, Nijkamp HJJ, Hille J: Identification and isolation of the FEEBLY gene from tomato by transposon tagging. Mol Gen Genet 1996, 251:267-280.

27. Hamberg M, de Leon IP, Rodriguez MJ, Castresana C: alpha-Dioxygenases. Biochem Biophys Res Commun 2005, 338:169-174.

28. Bannenberg $G$, Martinez $M$, Rodriguez MJ, Lopez MA, de Leon IP, Hamberg M, Castresana C: Functional analysis of $\alpha-D O X 2$, an active $\alpha$-dioxygenase critical for normal development in tomato plants. Plant Physiol 2009, 109.145094.

29. Halitschke R, Gase K, Hui DQ, Schmidt DD, Baldwin IT: Molecular interactions between the specialist herbivore Manduca sexta (Lepidoptera, Sphingidae) and its natural host Nicotiana attenuata. VI. Microarray analysis reveals that most herbivore-specific transcriptional changes are mediated by fatty acid-amino acid conjugates. Plant Physiol 2003, 131:1894-1902

30. Hui D, lqbal J, Lehmann K, Gase K, Saluz HP, Baldwin IT: Molecular interactions between the specialist herbivore Manduca sexta (Lepidoptera, Sphingidae) and its natural host Nicotiana attenuata: V. Microarray analysis and further characterization of large-scale changes in herbivore-induced mRNAs. Plant Physiol 2003, 131:1877-1893.

31. Gould K, Davies K, Winefield C, (Eds): Anthocyanins: Biosynthesis, Functions, and Applications. New York: Springer 2009.

32. Stevens MA, Rick CM: Genetics and breeding. The tomato crop, a scientific basis for crop improvement Chapman and Hall, New YorkAtherton JG, Rudich J 1986, 35-100.

33. Zhou XF, Hua DP, Chen ZZ, Zhou ZJ, Gong ZZ: Elongator mediates ABA responses, oxidative stress resistance and anthocyanin biosynthesis in Arabidopsis. Plant J 2009, 60:79-90.

34. Vellosillo T, Martinez M, Lopez MA, Vicente J, Cascon T, Dolan L, Hamberg M, Castresana C: Oxylipins produced by the 9-lipoxygenase pathway in Arabidopsis regulate lateral root development and defense responses through a specific signaling cascade. Plant Cell 2007, 19:831-846.

35. Khosravi D, Yang ECC, Siu KWM, Shore JS: High level of alpha-dioxygenase in short styles of distylous Turnera species. International Journal of Plant Sciences 2004, 165:995-1006.
36. Ikeda N, Ishihara M, Tsuneya T, Kawakita M, Yoshihara M, Suzuki Y, Komaki R, Inui M: Volatile components of honeysuckle (Lonicera japonica thunb.) flowers. Flavour and Fragrance Journal 1994, 9:325-331.

37. Krügel T, Lim M, Gase K, Halitschke R, Baldwin IT: Agrobacterium-mediated transformation of Nicotiana attenuata, a model ecological expression system. Chemoecology 2002, 12:177-183.

38. Bubner B, Gase K, Berger B, Link D, Baldwin IT: Occurrence of tetraploidy in Nicotiana attenuata plants after Agrobacterium-mediated transformation is genotype specific but independent of polysomaty of explant tissue. Plant Cell Reports 2006, 25:668-675.

39. Saedler R, Baldwin IT: Virus-induced gene silencing of jasmonate-induced direct defences, nicotine and trypsin proteinase-inhibitors in Nicotiana attenuata. J Exp Bot 2004, 55:151-157.

40. Kohlmann M, Bachmann A, Weichert H, Kolbe A, Balkenhohl T, Wasternack C, Feussner I: Formation of lipoxygenase-pathway-derived aldehydes in barley leaves upon methyl jasmonate treatment. European Journal of Biochemistry 1999, 260:885-895.

41. Wu JQ, Hettenhausen C, Meldau S, Baldwin IT: Herbivory rapidly activates MAPK signaling in attacked and unattacked leaf regions but not between leaves of Nicotiana attenuata. Plant Cell 2007, 19:1096-1122.

42. Rabino I, Mancinelli AL: Light, temperature, and anthocyanin production. Plant Physiol 1986, 81:922-924.

doi:10.1186/1471-2229-10-171

Cite this article as: Steppuhn et al:: The two $\alpha$-dox genes of Nicotiana attenuata: overlapping but distinct functions in development and stress responses. BMC Plant Biology 2010 10:171

\section{Submit your next manuscript to BioMed Central and take full advantage of:}

- Convenient online submission

- Thorough peer review

- No space constraints or color figure charges

- Immediate publication on acceptance

- Inclusion in PubMed, CAS, Scopus and Google Scholar

- Research which is freely available for redistribution
Biomed Central 